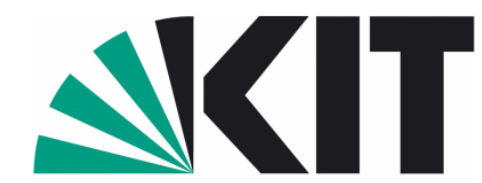

Karlsruher Institut für Technologie

\title{
A Primal-Dual Finite Element Approximation For A Nonlocal Model in Plasticity
}

\author{
C. Wieners \\ B. Wohlmuth
}

Preprint Nr.10/05

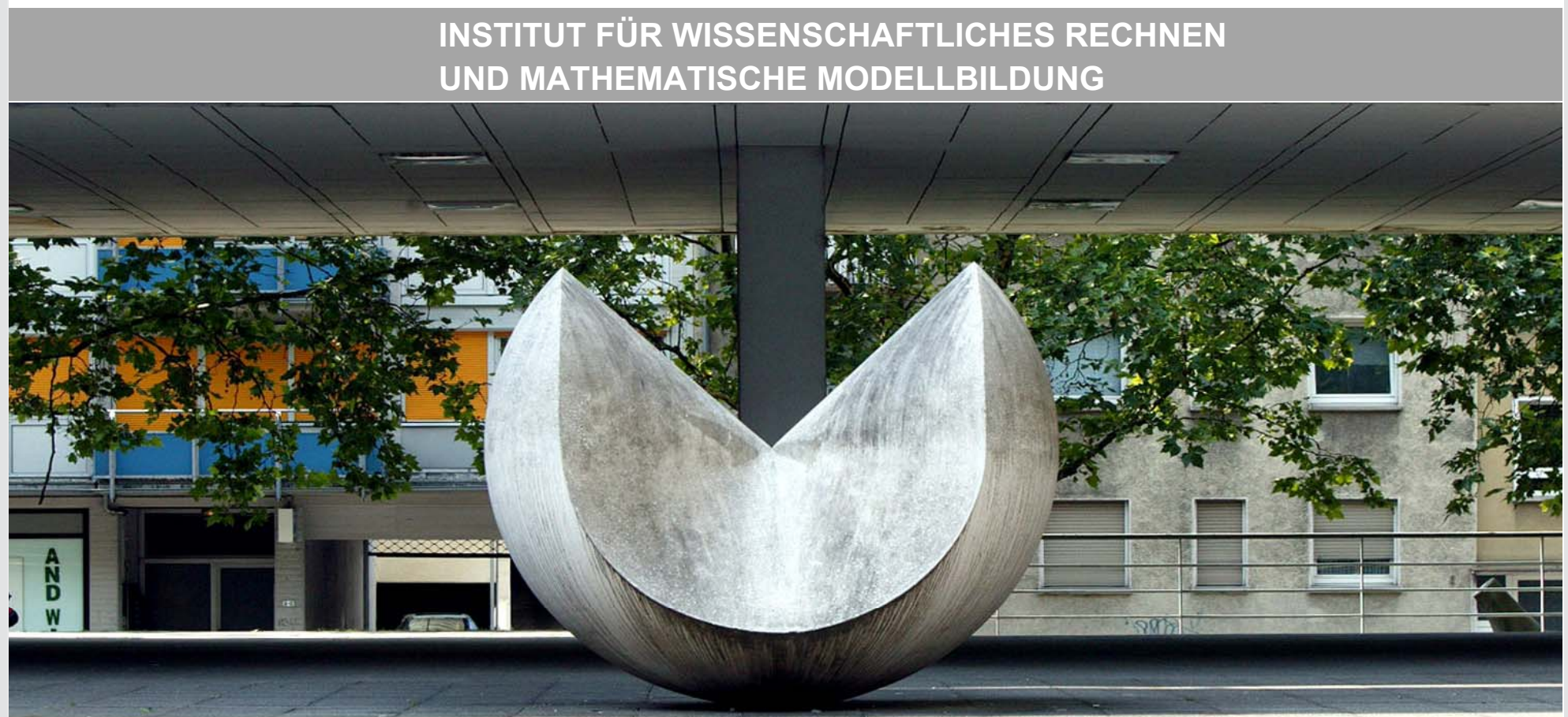

KIT - Universität des Landes Baden-Württemberg und

nationales Forschungszentrum in der Helmholtz-Gemeinschaft 


\section{Anschriften der Verfasser:}

Prof. Dr. Christian Wieners

Institut für Angewandte und Numerische Mathematik

Karlsruher Institut für Technologie (KIT)

D-76128 Karlsruhe

Prof. Dr. Barbara Wohlmuth

Zentrum Mathematik M2

Technische Universität München

D-85386 Garching 


\title{
A PRIMAL-DUAL FINITE ELEMENT APPROXIMATION FOR A NONLOCAL MODEL IN PLASTICITY
}

\author{
C. WIENERS* AND B. WOHLMUTH ${ }^{\dagger}$
}

\begin{abstract}
We study the numerical approximation of a static infinitesimal plasticity model of kinematic hardening with a nonlocal extension involving the curl of the plastic variable. Here, the free energy to be minimized is a combination of the elastic energy and an additional term depending on the curl of the plastic variable. In a first step, we introduce the stress as dual variable and provide an equivalent primal-dual formulation resulting in a local flow rule. To obtain optimal a priori estimates, the finite element spaces have to satisfy a uniform inf-sup condition. Finally, we show that the associated nonlinear mixed formulation can be solved iteratively by a classical radial return algorithm. Numerical results illustrate the convergence of the applied discretization and the solver.
\end{abstract}

Key words. gradient plasticity, finite element estimates

AMS subject classifications. 65M12, 65M60, 65N22, 74H15, 74S05

1. Introduction. The abstract setting for variational inequalities provides a powerful framework for the analysis of infinitesimal plasticity and its finite element discretization, see [11] and the references therein. In the static case, the elastoplastic solution is determined by minimizing a (primal) functional for the displacement and the plastic variable. Unfortunately for perfect plasticity this functional is not uniformly convex, and the minimizer exists only in a weak sense. We refer to [28] where suitable Banach spaces for the displacement and the plastic variable are discussed. On the other hand, the idealistic model of perfect plasticity does not include hardening nor size effects reflecting internal length scales. There are different possibilities for including such effects, and in most cases this leads to a more regular model with an associated uniformly convex primal functional, and thus the theory of standard Sobolev spaces can be applied.

Here, we consider a class of nonlocal models which can be obtained by an extension of the classical plasticity model with kinematic hardening. More precisely, we study a subclass of gradient plasticity, where the corresponding elasto-plastic energy includes only the curl of the plastic strain, see, e.g., $[7,8,9,13,14,26,27]$ for the analytical, mechanical, and physical properties of such models. In $[17,18]$ it is shown that a representative nonlocal model of such type can also be transformed into a variational inequality.

The finite element analysis for variational inequalities in plasticity with kinematic hardening yields a priori estimates involving the best approximation error and additional nonconformity terms, see $[1,3]$. Here, we extend these results to our non-local formulation and work with curl-conforming finite elements for the plastic variable. A different approach for a discontinuous Galerkin formulation of a gradient plasticity model with full gradient terms is presented in $[5,6,20]$, and in [19] a discontinuous Galerkin approximation of a model containing the curl of the plastic strain is considered.

Following [11], we consider plasticity models which are completely determined by

${ }^{*}$ Institut für Angewandte und Numerische Mathematik, KIT, 76128 Karlsruhe, Germany. email: christian.wieners@kit.edu

${ }_{\dagger}$ Zentrum Mathematik M2, Technische Universität München, Boltzmannstr. 3, 85386 Garching, Germany. email: wohlmuth@ma.tum.de 
the free energy $W(\boldsymbol{u}, \boldsymbol{p})$ and a dissipation potential $j(\boldsymbol{p})$, where $\boldsymbol{u}$ denotes the displacement and $\boldsymbol{p}$ the plastic variable. In the static case, for a given load functional $\ell(\cdot)$ the elasto-plastic solution $(\boldsymbol{u}, \boldsymbol{p})$ is determined as minimizer of the primal functional

$$
J(\boldsymbol{u}, \boldsymbol{p})=W(\boldsymbol{u}, \boldsymbol{p})+j(\boldsymbol{p})-\ell(\boldsymbol{u})
$$

subject to essential boundary conditions. We assume, see, e.g., [7, 17, 20], that the free energy can be decomposed in an additive way into

$$
W(\boldsymbol{u}, \boldsymbol{p})=W_{\text {elastic }}\left(\varepsilon^{e}\right)+W_{\text {plastic }}(\boldsymbol{p}),
$$

where $\varepsilon^{e}=\varepsilon(\boldsymbol{u})-\operatorname{sym} \operatorname{dev} \boldsymbol{p}$ is the elastic strain. Moreover, for standard materials the plastic evolution is driven by the conjugate variables

$$
\begin{aligned}
\boldsymbol{\sigma} & =\partial W_{\text {elastic }}\left(\boldsymbol{\varepsilon}^{e}\right), \\
\boldsymbol{\beta} & =\partial W_{\text {plastic }}(\boldsymbol{p}) .
\end{aligned}
$$

In the infinitesimal case, linearized constitutive relations hold, i.e., $\sigma=C \varepsilon^{e}$ and $\boldsymbol{\beta}=D \boldsymbol{p}$, and we assume that the energy contributions are uniformly convex in $\varepsilon^{e}$ and $\boldsymbol{p}$, respectively. Then, the free energy has a primal, a dual, and a primal-dual representation:

$$
W(\boldsymbol{u}, \boldsymbol{p})=\left\langle C \boldsymbol{\varepsilon}^{e}, \boldsymbol{\varepsilon}^{e}\right\rangle+\langle D \boldsymbol{p}, \boldsymbol{p}\rangle=\left\langle\boldsymbol{\sigma}, C^{-1} \boldsymbol{\sigma}\right\rangle+\left\langle\boldsymbol{\beta}, D^{-1} \boldsymbol{\beta}\right\rangle=\left\langle\boldsymbol{\sigma}, \boldsymbol{\varepsilon}^{e}\right\rangle+\langle\boldsymbol{\beta}, \boldsymbol{p}\rangle .
$$

This allows us to reformulate the primal minimization problem as a dual minimization problem or equivalently as a mixed saddle point problem in terms of the primal and dual variables. For standard materials in infinitesimal plasticity, the analysis of the primal problem determining the displacement and the plastic variable $(\boldsymbol{u}, \boldsymbol{p})$ is well-established [11, Chap. 7]. The dual problem determining the generalized stress $(\boldsymbol{\sigma}, \boldsymbol{\beta})$ can be analyzed independently, see, e.g., [11, Chap. 8]. For local plasticity, the equivalence of both approaches is shown in [11, Th. 8.3]. A mixed approximation is considered in [21].

Here, we consider a representative model in gradient plasticity, where $W_{\text {plastic }}(\boldsymbol{p})$ depends on curl $\boldsymbol{p}$ and note that the back stress can also be represented in $L_{2}$. This observation gives rise to a local flow rule for the non-local model as it is the case for local plasticity.

The rest of this paper is organized as follows: In Section 2, the notation is introduced and different problem formulations are stated. For the numerical simulation, we propose in Section 3 a mixed discretization scheme which approximates the primal and the dual solution. In Section 4, we provide a priori estimates. To obtain order $h$ bounds for the discretization error in the stress and the plastic variable, a uniform infsup condition has to be satisfied or alternatively an order $h^{2}$ best approximation error in the $L^{2}$-norm has to hold. Section 5 is devoted to the numerical solver. The mixed formulation is of special interest for the construction of a locally defined semi-smooth Newton which turns out to be equivalent to the classical radial return mapping. Finally, numerical results are presented in Section 6 illustrating the performance of the algorithm.

2. A model for infinitesimal nonlocal plasticity with hardening. Let the reference configuration $\Omega$ be a bounded Lipschitz domain in $\mathbf{R}^{3}$, and let $\Gamma_{D} \cup \Gamma_{N}=$ $\partial \Omega$ be a non-overlapping decomposition of the boundary, where we assume that $\Gamma_{D}$ has a positive 2-d measure. For simplicity of notation, we only consider the case of homogeneous Dirichlet boundary conditions. We define the spaces $\boldsymbol{V}=\{\boldsymbol{v} \in$ $\left.H^{1}\left(\Omega, \mathbf{R}^{3}\right):\left.\boldsymbol{v}\right|_{\Gamma_{D}}=\mathbf{0}\right\}$ for the displacements, $\boldsymbol{S}=L_{2}(\Omega, \operatorname{Sym}(3))$ for the stresses where $\operatorname{Sym}(3) \subset \mathbf{R}^{3 \times 3}$ denotes the set of symmetric tensors, and the space $\boldsymbol{Q}=\{\boldsymbol{q} \in$ $L_{2}\left(\Omega, \mathbf{R}^{3 \times 3}\right)$ : $\left.\operatorname{curl} \boldsymbol{q} \in L_{2}\left(\Omega, \mathbf{R}^{3 \times 3}\right)\right\}$ for the plastic variables. For tensors $\boldsymbol{q}=\left(q_{i j}\right)$, 
we set

$$
\operatorname{curl} \boldsymbol{q}=\left(\begin{array}{ccc}
\partial_{2} q_{13}-\partial_{3} q_{12} & \partial_{3} q_{11}-\partial_{1} q_{13} & \partial_{1} q_{12}-\partial_{2} q_{11} \\
\partial_{2} q_{23}-\partial_{3} q_{22} & \partial_{3} q_{21}-\partial_{1} q_{23} & \partial_{1} q_{22}-\partial_{2} q_{21} \\
\partial_{2} q_{33}-\partial_{3} q_{32} & \partial_{3} q_{31}-\partial_{1} q_{33} & \partial_{1} q_{32}-\partial_{2} q_{31}
\end{array}\right)
$$

and we use the weighted norms

$$
\begin{aligned}
\|\boldsymbol{v}\|_{\boldsymbol{V}}^{2} & =\int_{\Omega} \boldsymbol{\varepsilon}(\boldsymbol{v}): \mathbb{C}: \boldsymbol{\varepsilon}(\boldsymbol{v}) d \boldsymbol{x}, \quad \boldsymbol{v} \in \boldsymbol{V}, \\
\|\boldsymbol{\sigma}\|_{\boldsymbol{S}}^{2} & =\int_{\Omega} \boldsymbol{\sigma}: \mathbb{C}^{-1}: \boldsymbol{\sigma} d \boldsymbol{x}, \quad \boldsymbol{\sigma} \in \boldsymbol{S}, \\
\|\boldsymbol{q}\|_{\boldsymbol{Q}}^{2} & =\int_{\Omega} \boldsymbol{q}: \mathbb{H}: \boldsymbol{q} d \boldsymbol{x}+\int_{\Omega} \operatorname{curl} \boldsymbol{q}: \mathbb{L}: \operatorname{curl} \boldsymbol{q} d \boldsymbol{x}, \quad \boldsymbol{q} \in \boldsymbol{Q} .
\end{aligned}
$$

Here, $\mathbb{C}: \operatorname{Sym}(3) \longrightarrow \operatorname{Sym}(3)$ defined by $\mathbb{C}: \varepsilon=2 \mu \varepsilon+\lambda \operatorname{trace}(\varepsilon) \boldsymbol{I}$ is the isotropic elasticity tensor depending on the Lamé constants $\lambda, \mu>0, \mathbb{H}: \boldsymbol{q}=H_{0} \boldsymbol{q}$ measures the kinematic hardening, where $H_{0}>0$ is the kinematic hardening modulus, and $\mathbb{L}$ : $\operatorname{curl} \boldsymbol{q}=\mu L_{0}^{2} \operatorname{curl} \boldsymbol{q}$ describes the nonlocal energy contribution. Here $L_{0}>0$ is a length scale parameter. The linearized strain tensor is denoted by $\varepsilon(\boldsymbol{v})=\operatorname{sym}(D \boldsymbol{v})$ with $\operatorname{sym}(\boldsymbol{q})=\frac{1}{2}\left(\boldsymbol{q}+\boldsymbol{q}^{T}\right)$. Finally, the deviatoric part is given by $\operatorname{dev} \boldsymbol{\varepsilon}=\boldsymbol{\varepsilon}-\frac{1}{3} \operatorname{trace}(\varepsilon) \boldsymbol{I}$.

Note that $\|\cdot\|_{\boldsymbol{V}}$ is a norm on $\boldsymbol{V}$ due to Korn's inequality. We use $\|\cdot\|_{s, \omega}$ and $(\cdot, \cdot)_{s, \omega}$ for the norm and the inner product in $H^{s}\left(\omega, \mathbf{R}^{3 \times 3}\right), \omega \subset \Omega, s \geq 0$, respectively, and we drop the index $\omega$ in the case that $\omega=\Omega$. The inner product in $\boldsymbol{Q}$ is denoted by $(\cdot, \cdot)_{\boldsymbol{Q}}$, and $\boldsymbol{Q}^{\prime}$ stands for the dual space of $\boldsymbol{Q}$. Moreover $0<c, C<\infty$ are generic constants possibly depending on the material parameters but not on the mesh size of the discretization.

2.1. The free energy and the primal problem. In our model, we consider the free energy given by

$$
W(\boldsymbol{u}, \boldsymbol{p})=W_{\text {elastic }}\left(\varepsilon^{e}\right)+W_{\text {plastic }}(\boldsymbol{p})=\frac{1}{2} \int_{\Omega} \varepsilon^{e}: \mathbb{C}: \boldsymbol{\varepsilon}^{e} d \boldsymbol{x}+\frac{1}{2}\|\boldsymbol{p}\|_{\boldsymbol{Q}}^{2},
$$

where $\varepsilon^{e}=\boldsymbol{\varepsilon}(\boldsymbol{u})-\varepsilon^{p}$ is the elastic strain and $\varepsilon^{p}=$ sym dev $\boldsymbol{p}$ is the plastic strain. The plastic strain $\varepsilon^{p}$ is in the space $\boldsymbol{E}=\left\{\boldsymbol{\eta} \in L_{2}(\Omega, \operatorname{Sym}(3))\right.$ : trace $\left.\boldsymbol{\eta}=\mathbf{0}\right\}$. In $\boldsymbol{E}$, we use the weighted norm $\|\boldsymbol{\eta}\|_{\boldsymbol{E}}^{2}=(\mathbb{H}: \boldsymbol{\eta}, \boldsymbol{\eta})_{0}$. The dual space of $\boldsymbol{E}$ will be denoted by $\boldsymbol{B}$, i.e., $\boldsymbol{B}=\left\{\boldsymbol{\delta} \in L_{2}(\Omega\right.$, $\operatorname{Sym}(3))$ : trace $\left.\boldsymbol{\delta}=0\right\}=\boldsymbol{E}^{\prime}$. In $\boldsymbol{B}$, we use the weighted norm $\|\boldsymbol{\delta}\|_{\boldsymbol{B}}^{2}=\left(\mathbb{H}^{-1}: \boldsymbol{\delta}, \boldsymbol{\delta}\right)$.

The free energy defines the conjugate variables $\boldsymbol{\sigma}=C \boldsymbol{\varepsilon}^{e} \in \boldsymbol{S}$ and $\boldsymbol{\beta}=D \boldsymbol{p} \in \boldsymbol{Q}^{\prime}$. Here the operator $C: L_{2}(\Omega, \operatorname{Sym}(3)) \longrightarrow S$ is defined locally by $\boldsymbol{\sigma}=\mathbb{C}: \boldsymbol{\varepsilon}^{e}$, and $D: \boldsymbol{Q} \longrightarrow \boldsymbol{Q}^{\prime}$ is the non-local duality mapping defined by $\langle D \boldsymbol{p}, \boldsymbol{q}\rangle=(\boldsymbol{p}, \boldsymbol{q})_{\boldsymbol{Q}}$ for all $\boldsymbol{q} \in \boldsymbol{Q}$.

We start with the primal formulation in the space $\boldsymbol{Y}=\boldsymbol{V} \times \boldsymbol{Q}$ with norm $\|\boldsymbol{y}\|_{\boldsymbol{Y}}^{2}=$ $\|\boldsymbol{v}\|_{\boldsymbol{V}}^{2}+\|\boldsymbol{q}\|_{\boldsymbol{Q}}^{2}$ for $\boldsymbol{y}=(\boldsymbol{v}, \boldsymbol{q}) \in \boldsymbol{Y}$ and consider on $\boldsymbol{Y} \times \boldsymbol{Y}$ the bilinear form

and the convex functional

$$
a(\boldsymbol{y}, \tilde{\boldsymbol{y}})=\int_{\Omega}(\varepsilon(\boldsymbol{v})-\operatorname{sym} \operatorname{dev} \boldsymbol{q}): \mathbb{C}:(\varepsilon(\tilde{\boldsymbol{v}})-\operatorname{sym} \operatorname{dev} \tilde{\boldsymbol{q}}) d \boldsymbol{x}+(\boldsymbol{q}, \tilde{\boldsymbol{q}})_{\boldsymbol{Q}}
$$

$$
j(\boldsymbol{q})=\int_{\Omega} K_{0}|\operatorname{sym} \operatorname{dev} \boldsymbol{q}| d \boldsymbol{x}, \quad \boldsymbol{q} \in L_{2}\left(\Omega, \mathbf{R}^{3 \times 3}\right),
$$

where $K_{0}>0$ is the yield stress and $|\boldsymbol{q}|=\sqrt{\boldsymbol{q}: \boldsymbol{q}}$.

Depending on a body force density $\boldsymbol{b} \in L_{2}\left(\Omega, \mathbf{R}^{3}\right)$ and a traction force density $\boldsymbol{t}_{N} \in L_{2}\left(\Gamma_{N}, \mathbf{R}^{3}\right)$, we define the load functional $\ell \in \boldsymbol{V}^{\prime}$ by

$$
\ell(\boldsymbol{v})=\int_{\Omega} \boldsymbol{b} \cdot \boldsymbol{v} d \boldsymbol{x}+\int_{\Gamma_{N}} \boldsymbol{t}_{N} \cdot \boldsymbol{v} d \boldsymbol{a}, \quad \boldsymbol{v} \in \boldsymbol{V} .
$$


Lemma 2.1. A unique minimizer $\boldsymbol{z}=(\boldsymbol{u}, \boldsymbol{p}) \in \boldsymbol{Y}$ of

$$
J(\boldsymbol{z})=\frac{1}{2} a(\boldsymbol{z}, \boldsymbol{z})+j(\boldsymbol{p})-\ell(\boldsymbol{u})
$$

exists. The minimizer is uniquely characterized by the variational inequality

$$
a(\boldsymbol{z}, \boldsymbol{y}-\boldsymbol{z})+j(\boldsymbol{q})-j(\boldsymbol{p}) \geq \ell(\boldsymbol{v}-\boldsymbol{u}), \quad \boldsymbol{y}=(\boldsymbol{v}, \boldsymbol{q}) \in \boldsymbol{Y} .
$$

Proof. Obviously, all involved functionals are continuous. Moreover, $j(\cdot)$ is convex. Then Lemma 2.1 results from [11, Th. 6.6] provided the bilinear form $a(\cdot, \cdot)$ is $\boldsymbol{Y}$-elliptic. Observing that $\mathbb{C}^{-1} \operatorname{sym} \operatorname{dev} \boldsymbol{q}=1 /(2 \mu)$ sym $\operatorname{dev} \boldsymbol{q}$ and $|\operatorname{sym} \operatorname{dev} \boldsymbol{q}| \leq|\boldsymbol{q}|$, we find for $\boldsymbol{y} \in \boldsymbol{Y}$ and $s>1$

$$
\begin{aligned}
a(\boldsymbol{y}, \boldsymbol{y}) & =\|\mathbb{C}: \boldsymbol{\varepsilon}(\boldsymbol{v})-2 \mu \operatorname{sym} \operatorname{dev} \boldsymbol{q}\|_{\boldsymbol{S}}^{2}+\|\boldsymbol{q}\|_{\boldsymbol{Q}}^{2} \\
& \geq\left(1-\frac{1}{s}\right)\|\mathbb{C}: \boldsymbol{\varepsilon}(\boldsymbol{v})\|_{\boldsymbol{S}}^{2}+(1-s)\|2 \mu \mathrm{sym} \operatorname{dev} \boldsymbol{q}\|_{\boldsymbol{S}}^{2}+\|\boldsymbol{q}\|_{\boldsymbol{Q}}^{2} \\
& \geq \frac{s-1}{s}\|\boldsymbol{v}\|_{\boldsymbol{V}}^{2}-\frac{2 \mu(s-1)}{H_{0}}\|\boldsymbol{q}\|_{\boldsymbol{Q}}^{2}+\|\boldsymbol{q}\|_{\boldsymbol{Q}}^{2} \\
& \geq \min \left\{\frac{s-1}{s}, 1-\frac{2 \mu(s-1)}{H_{0}}\right\}\|\boldsymbol{y}\|_{\boldsymbol{Y}}^{2} .
\end{aligned}
$$

The lower bound holds for all $s>1$ yielding the ellipticity constant

$\square$

$$
c_{0}=\max _{s>1} \min \left\{\frac{s-1}{s}, 1-\frac{2 \mu(s-1)}{H_{0}}\right\}=\frac{H_{0}}{\mu+H_{0}+\sqrt{\mu^{2}+2 \mu H_{0}}} .
$$

2.2. The primal-dual problem. The primal solution $(\boldsymbol{u}, \boldsymbol{p})$ defines the plastic strain $\varepsilon^{p}=\operatorname{sym} \operatorname{dev} \boldsymbol{p}$ and and the conjugated variables $\boldsymbol{\sigma}=\mathbb{C}:\left(\varepsilon(\boldsymbol{u})-\varepsilon^{p}\right)$ and $\boldsymbol{\beta}$. Here, the plastic variable $\boldsymbol{p} \in \boldsymbol{Q}$ and the conjugate variable $\boldsymbol{\beta} \in \boldsymbol{Q}^{\prime}$ can be identified by the Riesz representation theorem. Testing the variational inequality (2.2) with $\boldsymbol{y}=(\boldsymbol{u} \pm \boldsymbol{v}, \boldsymbol{p})$ and $\boldsymbol{y}=(\boldsymbol{u}, \boldsymbol{q})$ results in a weak equilibrium equation

$$
\int_{\Omega} \boldsymbol{\sigma}: \boldsymbol{\varepsilon}(\boldsymbol{v}) d \boldsymbol{x}=\ell(\boldsymbol{v}), \quad \boldsymbol{v} \in \boldsymbol{V}
$$

and the dissipation inequality for the plastic variable

$$
j(\boldsymbol{q}) \geq j(\boldsymbol{p})+(\operatorname{dev} \boldsymbol{\sigma}, \boldsymbol{q}-\boldsymbol{p})_{0}-(\boldsymbol{p}, \boldsymbol{q}-\boldsymbol{p})_{\boldsymbol{Q}}, \quad \boldsymbol{q} \in \boldsymbol{Q} .
$$

The following lemma shows that the back stress is more regular and lives in $\boldsymbol{B} \subset \boldsymbol{Q}^{\prime}$.

Lemma 2.2. Let $(\boldsymbol{u}, \boldsymbol{p}) \in \boldsymbol{V} \times \boldsymbol{Q}$ be the solution of (2.2). Then, there exists a unique $\boldsymbol{\beta} \in \boldsymbol{B}$ such that

$$
(\boldsymbol{\beta}, \boldsymbol{q})_{0}=(\boldsymbol{p}, \boldsymbol{q})_{\boldsymbol{Q}}, \quad \boldsymbol{q} \in \boldsymbol{Q}
$$

Proof. Testing $(2.4 \mathrm{~b})$ with $\boldsymbol{p} \pm \boldsymbol{p}$ yields $0=j(\boldsymbol{p})-(\operatorname{dev} \boldsymbol{\sigma}, \boldsymbol{p})_{0}+(\boldsymbol{p}, \boldsymbol{p})_{\boldsymbol{Q}}$. Using the boundedness of $j(\cdot)$ in $\boldsymbol{E}$, we find in terms of $(2.4 \mathrm{~b})$

$$
\left|(\boldsymbol{p}, \boldsymbol{q})_{\boldsymbol{Q}}\right| \leq j(\boldsymbol{q})+|(\operatorname{dev} \boldsymbol{\sigma}, \boldsymbol{q})| \leq C(\boldsymbol{u}, \boldsymbol{p})\|\operatorname{sym} \operatorname{dev} \boldsymbol{q}\|_{\boldsymbol{E}}, \quad \boldsymbol{q} \in \boldsymbol{Q} .
$$

Thus the linear functional $(\boldsymbol{p}, \cdot)_{\boldsymbol{Q}} \in \boldsymbol{Q}^{\prime}$ is bounded in $\boldsymbol{E}$. Noting that $\boldsymbol{Q} \cap \boldsymbol{E}$ is dense in $\boldsymbol{E}$ and that $(\boldsymbol{p}, \boldsymbol{q})_{\boldsymbol{Q}}=0$ for $\operatorname{sym} \operatorname{dev} \boldsymbol{q}=\mathbf{0}$, there exists a unique $\boldsymbol{\beta} \in \boldsymbol{B}$ such that (2.5) holds. 
The higher regularity of $\boldsymbol{\beta}$ in combination with Lemma 2.1 yields the existence of a unique solution $(\boldsymbol{u}, \boldsymbol{p}, \boldsymbol{\beta}) \in \boldsymbol{V} \times \boldsymbol{Q} \times \boldsymbol{B}$ of the primal-dual problem

$$
\begin{array}{ll}
(\boldsymbol{\sigma}, \boldsymbol{\varepsilon}(\boldsymbol{v}))_{0}=\ell(\boldsymbol{v}), & \boldsymbol{v} \in \boldsymbol{V}, \\
(\boldsymbol{\beta}, \boldsymbol{q})_{0}=(\boldsymbol{p}, \boldsymbol{q})_{\boldsymbol{Q}}, & \boldsymbol{q} \in \boldsymbol{Q}, \\
j(\boldsymbol{\eta}) \geq j\left(\boldsymbol{\varepsilon}^{p}\right)+\left(\operatorname{dev} \boldsymbol{\sigma}-\boldsymbol{\beta}, \boldsymbol{\eta}-\boldsymbol{\varepsilon}^{p}\right)_{0}, & \boldsymbol{\eta} \in \boldsymbol{E} .
\end{array}
$$

Moreover the dissipation inequality (2.6c) is equivalent to the local flow rule

$$
\boldsymbol{\varepsilon}^{p}=\gamma \frac{\operatorname{dev} \boldsymbol{\sigma}-\boldsymbol{\beta}}{|\operatorname{dev} \boldsymbol{\sigma}-\boldsymbol{\beta}|}
$$

together with the KKT conditions

$$
|\operatorname{dev} \boldsymbol{\sigma}-\boldsymbol{\beta}| \leq K_{0}, \quad \gamma \geq 0, \quad \gamma\left(|\operatorname{dev} \boldsymbol{\sigma}-\boldsymbol{\beta}|-K_{0}\right)=0,
$$

see, e.g., [18, Sect. 2.3].

REMARK 2.3. The higher regularity of $\boldsymbol{\beta}$ implies an additional regularity on $\boldsymbol{p}$, i.e., curl curl $\boldsymbol{p} \in L_{2}\left(\Omega, \mathbf{R}^{3 \times 3}\right)$ and $\boldsymbol{\beta}=H_{0} \boldsymbol{p}+L_{0} \operatorname{curl} \operatorname{curl} \boldsymbol{p}$, see also [7, 17, 18], and thus the standard infinitesimal model of kinematic hardening is recovered in the limit $L_{0}$ tends to zero.

3. A finite element approximation of the primal-dual problem. In this section, we analyze the discretization error of the mixed problem. Associated with $\Omega$ is a shape regular family $\left\{\mathcal{T}_{h}\right\}_{h}$ of triangulations, the elements of which are affine equivalent tetrahedra or hexahedra. Let $\boldsymbol{V}_{h} \times \boldsymbol{Q}_{h} \subset \boldsymbol{V} \times \boldsymbol{Q}$ be a low order conforming finite element space for the primal solution with order $h$ best approximation properties with respect to the $\|\cdot\|_{\boldsymbol{Y}}$ norm. For the numerical simulation of models with curl-terms it is appropriate to use Nédélec elements. These types of elements are curl-conforming and well-established for the numerical approximation of Maxwell's equations [12, 16]. The plastic strain will be approximated in $\boldsymbol{E}_{h} \subset \boldsymbol{E}$. Here $\boldsymbol{E}_{h}$ stands for the space of symmetric trace free element-wise constant finite elements, and we denote by $\Pi_{h}$ the $L_{2}$-projection onto $\boldsymbol{E}_{h}$.

Our discrete primal-dual problem is then equivalent to a discrete primal problem defined by a mesh-dependent free energy. Introducing the mesh-dependent bilinear form on $\boldsymbol{Y}_{h}=\boldsymbol{V}_{h} \times \boldsymbol{Q}_{h}$

$a_{h}\left(\boldsymbol{y}_{h}, \tilde{\boldsymbol{y}}_{h}\right)=\left(\varepsilon\left(\boldsymbol{v}_{h}\right)-\Pi_{h} \operatorname{sym} \operatorname{dev} \boldsymbol{q}_{h}, \mathbb{C}:\left(\varepsilon\left(\tilde{\boldsymbol{v}}_{h}\right)-\Pi_{h} \operatorname{sym} \operatorname{dev} \tilde{\boldsymbol{q}}_{h}\right)\right)_{0}+\left(\boldsymbol{q}_{h}, \tilde{\boldsymbol{q}}_{h}\right)_{\boldsymbol{Q}}$ and the mesh-dependent convex functional $j_{h}\left(\boldsymbol{q}_{h}\right)=j\left(\Pi_{h} \boldsymbol{q}_{h}\right)$, we can define the discrete primal functional

$$
J_{h}\left(\boldsymbol{y}_{h}\right)=\frac{1}{2} a_{h}\left(\boldsymbol{y}_{h}, \boldsymbol{y}_{h}\right)+j_{h}\left(\boldsymbol{q}_{h}\right)-\ell\left(\boldsymbol{v}_{h}\right) .
$$

Using $\left\|\Pi_{h} \boldsymbol{q}\right\|_{0} \leq\left\|\boldsymbol{q}_{h}\right\|_{0}$, we find that the bilinear form $a_{h}(\cdot, \cdot)$ is also elliptic with the same ellipticity constant as $a(\cdot, \cdot)$. This observation guarantees that the solution $\left(\boldsymbol{u}_{h}, \boldsymbol{p}_{h}\right) \in \boldsymbol{V}_{h} \times \boldsymbol{Q}_{h}$ of the discrete equilibrium equation with $\boldsymbol{\sigma}_{h}=\mathbb{C}:\left(\varepsilon\left(\boldsymbol{u}_{h}\right)-\boldsymbol{\varepsilon}_{h}^{p}\right)$ and $\varepsilon_{h}^{p}=\Pi_{h} \operatorname{sym} \operatorname{dev} \boldsymbol{p}_{h}$

$$
\left(\boldsymbol{\sigma}_{h}, \boldsymbol{\varepsilon}\left(\boldsymbol{v}_{h}\right)\right)_{0}=\ell\left(\boldsymbol{v}_{h}\right), \quad \boldsymbol{v}_{h} \in \boldsymbol{V}_{h},
$$

and the dissipation inequality for the plastic variable

$$
j_{h}\left(\boldsymbol{q}_{h}\right) \geq j_{h}\left(\boldsymbol{p}_{h}\right)+\left(\operatorname{dev} \boldsymbol{\sigma}_{h}, \Pi_{h} \operatorname{sym} \operatorname{dev}\left(\boldsymbol{q}_{h}-\boldsymbol{p}_{h}\right)\right)_{0}-\left(\boldsymbol{p}_{h}, \boldsymbol{q}_{h}-\boldsymbol{p}_{h}\right)_{\boldsymbol{Q}}, \quad \boldsymbol{q}_{h} \in \boldsymbol{Q}_{h}
$$

is the unique minimizer of the discrete primal functional $J_{h}(\cdot)$ in $\boldsymbol{V}_{h} \times \boldsymbol{Q}_{h}$. 
As in the continuous setting, we can define a discrete back stress. To do so, we introduce $\boldsymbol{B}_{h}=\boldsymbol{E}_{h}$, and we define $\boldsymbol{B}_{h}^{0}=\boldsymbol{E}_{h}^{0}=\left\{\Pi_{h}\right.$ sym dev $\left.\boldsymbol{q}_{h}: \boldsymbol{q}_{h} \in \boldsymbol{Q}_{h}\right\} \subset \boldsymbol{E}_{h}$. From (3.2b), we observe that $\left(\boldsymbol{p}_{h}, \boldsymbol{q}_{h}\right)_{\boldsymbol{Q}}=0$ for all $\boldsymbol{q}_{h} \in \boldsymbol{Q}_{h}^{0}=\left\{\boldsymbol{q}_{h} \in \boldsymbol{Q}_{h}: \Pi_{h} \operatorname{sym} \operatorname{dev} \boldsymbol{q}_{h}=\right.$ $\mathbf{0}\}$, and thus there exists a unique solution $\left(\boldsymbol{u}_{h}, \boldsymbol{p}_{h}, \boldsymbol{\beta}_{h}\right) \in \boldsymbol{V}_{h} \times \boldsymbol{Q}_{h} \times \boldsymbol{B}_{h}^{0}$ of the discrete primal-dual problem

$$
\begin{array}{ll}
\left(\boldsymbol{\sigma}_{h}, \boldsymbol{\varepsilon}\left(\boldsymbol{v}_{h}\right)\right)_{0}=\ell\left(\boldsymbol{v}_{h}\right), & \boldsymbol{v}_{h} \in \boldsymbol{V}_{h}, \\
\left(\boldsymbol{\beta}_{h}, \boldsymbol{q}_{h}\right)_{0}=\left(\boldsymbol{p}_{h}, \boldsymbol{q}_{h}\right)_{\boldsymbol{Q}}, & \boldsymbol{q}_{h} \in \boldsymbol{Q}_{h}, \\
j\left(\boldsymbol{\eta}_{h}\right) \geq j\left(\varepsilon_{h}^{p}\right)+\left(\operatorname{dev} \boldsymbol{\sigma}_{h}-\boldsymbol{\beta}_{h}, \boldsymbol{\eta}_{h}-\varepsilon_{h}^{p}\right)_{0}, & \boldsymbol{\eta}_{h} \in \boldsymbol{E}_{h}^{0} .
\end{array}
$$

REMARK 3.1. In the case $\boldsymbol{E}_{h}=\boldsymbol{E}_{h}^{0}$, we can use locally constant basis functions in the discrete dissipation inequality (3.3c) resulting in the following point-wise flow rule

$$
\varepsilon_{h}^{p}=\gamma_{h} \frac{\Pi_{h} \operatorname{dev} \boldsymbol{\sigma}_{h}-\boldsymbol{\beta}_{h}}{\left|\Pi_{h} \operatorname{dev} \boldsymbol{\sigma}_{h}-\boldsymbol{\beta}_{h}\right|}
$$

with the return parameter $\gamma_{h}=\left|\varepsilon_{h}^{p}\right|$ characterized by the local KKT conditions

$$
\left|\Pi_{h} \operatorname{dev} \boldsymbol{\sigma}_{h}-\boldsymbol{\beta}_{h}\right| \leq K_{0}, \quad \gamma_{h} \geq 0, \quad \gamma_{h}\left(\left|\Pi_{h} \operatorname{dev} \boldsymbol{\sigma}_{h}-\boldsymbol{\beta}_{h}\right|-K_{0}\right)=0 .
$$

Thus, the classical radial return algorithm can be applied.

It is easy to see that $\boldsymbol{B}_{h}=\boldsymbol{B}_{h}^{0}$ and thus $\boldsymbol{E}_{h}=\boldsymbol{E}_{h}^{0}$ if the pair $\left(\boldsymbol{Q}_{h}, \boldsymbol{B}_{h}\right)$ satisfies a uniform inf-sup stability, i.e., there exists a constant $c_{\text {stab }}>0$ independent of the mesh size such that

$$
\sup _{\boldsymbol{q}_{h} \in \boldsymbol{Q}_{h}, \boldsymbol{q}_{h} \neq 0} \frac{\left(\boldsymbol{\delta}_{h}, \boldsymbol{q}_{h}\right)_{0}}{\left\|\boldsymbol{q}_{h}\right\|_{\boldsymbol{Q}}} \geq c_{\text {stab }}\left\|\boldsymbol{\delta}_{h}\right\|_{\boldsymbol{Q}^{\prime}}, \quad \boldsymbol{\delta}_{h} \in \boldsymbol{B}_{h}
$$

However, the uniform inf-sup stability of the pair $\left(\boldsymbol{Q}_{h}, \boldsymbol{B}_{h}\right)$ is not a necessary condition for having $\boldsymbol{B}_{h}=\boldsymbol{B}_{h}^{0}$. For each given $\boldsymbol{Q}_{h}$, it is easy to construct an enriched space in terms of locally defined element bubbles $b_{T}$ with $b_{T} \geq 0$, supp $b_{T}=\bar{T}, h\left\|\nabla b_{T}\right\|_{0, T} \leq$ $C\left\|b_{T}\right\|_{0, T}$. Adding $\prod_{T \in \mathcal{T}_{h}} \operatorname{span}\left\{b_{T} \varepsilon_{i}: i=1, \ldots, 5\right\}$, where $\varepsilon_{1}, \ldots, \varepsilon_{5}$ is an orthogonal basis for $\operatorname{sl}(3) \cap \operatorname{Sym}(3)$, to $\boldsymbol{Q}_{h}$, it is easy to see that the enriched space satisfies $\boldsymbol{B}_{h}=$ $\boldsymbol{B}_{h}^{0}$. Moreover, these additional degrees of freedom can easily be eliminated locally, and we have to solve a non-linear system of the original dimension and complexity. Thus from now on, we always assume without loss of generality that $\boldsymbol{B}_{h}=\boldsymbol{B}_{h}^{0}$.

4. A priori discretization error estimates for the primal-dual problem. In this section, we provide a priori estimates for the primal-dual problem. As we will see, order $h$ results can be only obtained under some additional assumptions. Here, we discuss two cases separately. The case that $\boldsymbol{Q}_{h}$ satisfies an order $h^{2}$ best approximation property in the $L^{2}$-norm and the case that the pair $\left(\boldsymbol{Q}_{h}, \boldsymbol{B}_{h}\right)$ satisfies a uniform inf-sup stability.

4.1. Error estimates for the primal solutions. The following theorem shows that the error in the stress and the plastic variable can be bounded by the best approximation error and the influence of the error in the back stress on the plastic variable.

ThEOREM 4.1. There exists a constant $C<\infty$ independent of the mesh size but possibly depending on the material parameters such that

$$
\begin{aligned}
\left\|\boldsymbol{\sigma}-\boldsymbol{\sigma}_{h}\right\|_{\boldsymbol{S}}^{2}+\left\|\boldsymbol{p}-\boldsymbol{p}_{h}\right\|_{\boldsymbol{Q}}^{2} \leq & C\left(\inf _{\boldsymbol{v}_{h} \in \boldsymbol{V}_{h}}\left\|\boldsymbol{u}-\boldsymbol{v}_{h}\right\|_{\boldsymbol{V}}^{2}+\inf _{\boldsymbol{\delta}_{h} \in \boldsymbol{B}_{h}}\left\|\boldsymbol{\beta}-\boldsymbol{\delta}_{h}\right\|_{\boldsymbol{B}}^{2}\right. \\
& \left.+\inf _{\boldsymbol{q}_{h} \in \boldsymbol{Q}_{h}}\left(\left\|\boldsymbol{p}-\boldsymbol{q}_{h}\right\|_{\boldsymbol{Q}}^{2}+\left|\left(\boldsymbol{\beta}-\boldsymbol{\beta}_{h}, \boldsymbol{q}_{h}-\boldsymbol{p}\right)_{0}\right|\right)\right) .
\end{aligned}
$$


Proof. We start with an upper bound for the error in the stress. Using the Galerkin orthogonality $\left(\boldsymbol{\sigma}-\boldsymbol{\sigma}_{h}, \boldsymbol{\varepsilon}\left(\boldsymbol{v}_{h}\right)\right)_{0}=0$ for $\boldsymbol{v}_{h} \in \boldsymbol{V}_{h}$, we find

$$
\begin{aligned}
\left\|\boldsymbol{\sigma}-\boldsymbol{\sigma}_{h}\right\|_{\boldsymbol{S}}^{2} & =\left(\boldsymbol{\sigma}-\boldsymbol{\sigma}_{h}, \boldsymbol{\varepsilon}\left(\boldsymbol{u}-\boldsymbol{u}_{h}\right)\right)_{0}-\left(\boldsymbol{\sigma}-\boldsymbol{\sigma}_{h}, \boldsymbol{\varepsilon}^{p}-\varepsilon_{h}^{p}\right)_{0} \\
& \leq\left\|\boldsymbol{\sigma}-\boldsymbol{\sigma}_{h}\right\|_{\boldsymbol{S}} \inf _{\boldsymbol{v}_{h} \in \boldsymbol{V}_{h}}\left\|\boldsymbol{u}-\boldsymbol{v}_{h}\right\|_{\boldsymbol{V}}+\left(\operatorname{dev} \boldsymbol{\sigma}-\operatorname{dev} \boldsymbol{\sigma}_{h}, \varepsilon_{h}^{p}-\boldsymbol{\varepsilon}^{p}\right)_{0} .
\end{aligned}
$$

To bound the second term on the right side, we set $\boldsymbol{\eta}=\varepsilon_{h}^{p}$ in (2.6c) and $\boldsymbol{\eta}_{h}=\Pi_{h} \varepsilon^{p}$ in (3.3c). Adding both inequalities and using $j\left(\Pi_{h} \varepsilon^{p}\right) \leq j\left(\varepsilon^{p}\right)$, we get

$$
\left(\operatorname{dev} \boldsymbol{\sigma}, \varepsilon_{h}^{p}-\varepsilon^{p}\right)_{0}+\left(\operatorname{dev} \boldsymbol{\sigma}_{h}, \Pi_{h} \varepsilon^{p}-\varepsilon_{h}^{p}\right)_{0} \leq\left(\boldsymbol{\beta}-\boldsymbol{\beta}_{h}, \varepsilon_{h}^{p}-\varepsilon^{p}\right)_{0} .
$$

In a second step, we consider the error in the plastic variable. Exploiting (2.6b) and (3.3b), we obtain for all $\boldsymbol{q}_{h} \in \boldsymbol{Q}_{h}$

$$
\left\|\boldsymbol{p}-\boldsymbol{p}_{h}\right\|_{\boldsymbol{Q}}^{2} \leq\left\|\boldsymbol{p}-\boldsymbol{p}_{h}\right\|_{\boldsymbol{Q}}\left\|\boldsymbol{p}-\boldsymbol{q}_{h}\right\|_{\boldsymbol{Q}}+\left(\boldsymbol{\beta}-\boldsymbol{\beta}_{h}, \boldsymbol{q}_{h}-\boldsymbol{p}_{h}\right)_{0}
$$

Combing the second term on the right side of (4.1) with the second term on the right side of (4.1), we find in terms of (4.2)

$$
\begin{aligned}
\left(\operatorname{dev} \boldsymbol{\sigma}-\operatorname{dev} \boldsymbol{\sigma}_{h}, \boldsymbol{\varepsilon}_{h}^{p}-\boldsymbol{\varepsilon}^{p}\right)_{0}+\left(\boldsymbol{\beta}-\boldsymbol{\beta}_{h}, \boldsymbol{q}_{h}-\boldsymbol{p}_{h}\right)_{0} \\
\quad \leq\left(\operatorname{dev} \boldsymbol{\sigma}_{h}, \boldsymbol{p}-\Pi_{h} \boldsymbol{p}\right)_{0}+\left(\boldsymbol{\beta}-\boldsymbol{\beta}_{h}, \boldsymbol{q}_{h}-\boldsymbol{p}\right)_{0}+\left(\boldsymbol{\beta}, \Pi_{h} \boldsymbol{p}_{h}-\boldsymbol{p}_{h}\right)_{0} .
\end{aligned}
$$

The first term is bounded for all $\boldsymbol{q}_{h} \in \boldsymbol{Q}_{h}$ by

$\left(\operatorname{dev} \boldsymbol{\sigma}_{h}, \boldsymbol{p}-\Pi_{h} \boldsymbol{p}\right)_{0} \leq 2\left\|\operatorname{dev}\left(\boldsymbol{\sigma}-\boldsymbol{\sigma}_{h}\right)\right\|_{\boldsymbol{B}}\left\|\varepsilon^{p}-\Pi_{h} \varepsilon^{p}\right\|_{\boldsymbol{E}} \leq 4\left\|\boldsymbol{\sigma}-\boldsymbol{\sigma}_{h}\right\|_{\boldsymbol{B}}\left\|\boldsymbol{p}-\boldsymbol{q}_{h}\right\|_{\boldsymbol{Q}}$, and for the last term, we obtain for all $\boldsymbol{\delta}_{h} \in \boldsymbol{B}_{h}$

$$
\left(\boldsymbol{\beta}, \Pi_{h} \boldsymbol{p}_{h}-\boldsymbol{p}_{h}\right)_{0} \leq 2\left\|\boldsymbol{\beta}-\boldsymbol{\delta}_{h}\right\|_{\boldsymbol{B}}\left\|\boldsymbol{p}-\boldsymbol{p}_{h}\right\|_{\boldsymbol{E}} \leq 2\left\|\boldsymbol{\beta}-\boldsymbol{\delta}_{h}\right\|_{\boldsymbol{B}}\left\|\boldsymbol{p}-\boldsymbol{p}_{h}\right\|_{\boldsymbol{Q}} .
$$

In a next step, we add (4.1) and (4.1) and use the last inequality to get

$$
\begin{aligned}
\left\|\boldsymbol{\sigma}-\boldsymbol{\sigma}_{h}\right\|_{\boldsymbol{S}}^{2}+\left\|\boldsymbol{p}-\boldsymbol{p}_{h}\right\|_{\boldsymbol{Q}}^{2} \leq & \left\|\boldsymbol{\sigma}-\boldsymbol{\sigma}_{h}\right\|_{\boldsymbol{S}} \inf _{\boldsymbol{v}_{h} \in \boldsymbol{V}_{h}}\left\|\boldsymbol{u}-\boldsymbol{v}_{h}\right\|_{\boldsymbol{V}} \\
& +4\left\|\boldsymbol{\sigma}-\boldsymbol{\sigma}_{h}\right\|_{\boldsymbol{B}} \inf _{\boldsymbol{q}_{h} \in \boldsymbol{Q}_{h}}\left\|\boldsymbol{p}-\boldsymbol{q}_{h}\right\|_{\boldsymbol{Q}} \\
& +2\left\|\boldsymbol{p}-\boldsymbol{p}_{h}\right\|_{\boldsymbol{Q}} \inf _{\boldsymbol{\delta}_{h} \in \boldsymbol{B}_{h}}\left\|\boldsymbol{\beta}-\boldsymbol{\delta}_{h}\right\|_{\boldsymbol{B}} \\
& +\inf _{\boldsymbol{q}_{h} \in \boldsymbol{Q}_{h}}\left(\left\|\boldsymbol{p}-\boldsymbol{p}_{h}\right\|_{\boldsymbol{Q}}\left\|\boldsymbol{p}-\boldsymbol{q}_{h}\right\|_{\boldsymbol{Q}}+\left(\boldsymbol{\beta}-\boldsymbol{\beta}_{h}, \boldsymbol{q}_{h}-\boldsymbol{p}\right)_{0}\right) .
\end{aligned}
$$

Using $\left\|\boldsymbol{\sigma}-\boldsymbol{\sigma}_{h}\right\|_{\boldsymbol{B}} \leq C\left\|\boldsymbol{\sigma}-\boldsymbol{\sigma}_{h}\right\|_{\boldsymbol{S}}$ and Young's inequality, we find the assertion.

REMARK 4.2. The proof of Theorem 4.1 shows that the only term involving a constant depending on the parameter $H_{0}$ stems from $\left(\operatorname{dev} \boldsymbol{\sigma}_{h}, \boldsymbol{p}-\Pi_{h} \boldsymbol{p}\right)_{0}$. In the case of lowest order elements in $\boldsymbol{V}_{h}$ on tetrahedral meshes, we have $\operatorname{dev} \boldsymbol{\sigma}_{h}=\Pi_{h} \operatorname{dev} \boldsymbol{\sigma}_{h}$. As a consequence, we obtain in Theorem 4.1 a parameter independent bound.

If we consider the non-standard term $\left(\boldsymbol{\beta}-\boldsymbol{\beta}_{h}, \boldsymbol{q}_{h}-\boldsymbol{p}\right)_{0}$ in more detail, we find in terms of the triangle inequality, the KKT conditions (2.8) and (3.5)

$$
\left(\boldsymbol{\beta}-\boldsymbol{\beta}_{h}, \boldsymbol{q}_{h}-\boldsymbol{p}\right)_{0} \leq 2\left(K_{0} \sqrt{|\Omega|}+\left\|\boldsymbol{\sigma}-\boldsymbol{\sigma}_{h}\right\|_{0}\right)\left\|\boldsymbol{q}_{h}-\boldsymbol{p}\right\|_{0} .
$$

Observing $\left\|\boldsymbol{u}-\boldsymbol{u}_{h}\right\|_{\boldsymbol{V}} \leq\left\|\boldsymbol{\sigma}-\boldsymbol{\sigma}_{h}\right\|_{\boldsymbol{S}}+C\left\|\boldsymbol{p}-\boldsymbol{p}_{h}\right\|_{\boldsymbol{Q}}$ and using the best approximation properties of the spaces $\boldsymbol{V}_{h}, \boldsymbol{Q}_{h}$ and $\boldsymbol{B}_{h}$, we get the a priori estimate in terms of Theorem 4.1 and (4.3).

COROLlaRY 4.3. Under the regularity assumption $(\boldsymbol{u}, \boldsymbol{p}, \boldsymbol{\beta}) \in H^{3 / 2}\left(\Omega, \mathbf{R}^{3}\right) \times$ $H^{3 / 2}\left(\Omega, \mathbf{R}^{3 \times 3}\right) \times H^{1 / 2}\left(\Omega, \mathbf{R}^{3 \times 3}\right)$ on the solution, we find the sub-optimal a priori estimate

$$
\left\|\boldsymbol{u}-\boldsymbol{u}_{h}\right\|_{\boldsymbol{V}}+\left\|\boldsymbol{p}-\boldsymbol{p}_{h}\right\|_{\boldsymbol{Q}} \leq C \sqrt{h}\left(\|\boldsymbol{u}\|_{3 / 2}+\|\boldsymbol{p}\|_{3 / 2}+\|\boldsymbol{\beta}\|_{1 / 2}+1\right) .
$$

We remark that in contrast to Theorem 4.1, the constant in the upper bound depends for all type of meshes on the parameter and degenerates if $H_{0}$ tends to zero. 
4.2. An order $h$ a priori error estimate. In this subsection, we discuss two cases such that an order $h$ a priori estimate can be established.

Case 1: Order $h$ approximation property of $\boldsymbol{Q}_{h}$ in a mesh dependent norm

$$
\inf _{\boldsymbol{q}_{h} \in \boldsymbol{Q}_{h}}\left(\left\|\boldsymbol{q}-\boldsymbol{q}_{h}\right\|_{\boldsymbol{Q}}+h^{-1}\left\|\boldsymbol{q}-\boldsymbol{q}_{h}\right\|_{0}\right) \leq C h\|\boldsymbol{q}\|_{2}, \quad \boldsymbol{q} \in H^{2}\left(\Omega, \mathbf{R}^{3 \times 3}\right) .
$$

Case 2: Uniform inf-sup stability of the pair $\left(\boldsymbol{Q}_{h}, \boldsymbol{B}_{h}\right)$, i.e., (3.6) is satisfied with a mesh independent constant.

Before we are going to establish our main result, we discuss briefly two examples of finite element spaces satisfying Case 1 or Case 2.

Unfortunately, Case 1 does not hold for lowest order Nédélec elements. However Case 1 is satisfied for second order Nédélec elements, cf. [15, Th. 5.41], or for the lowest order curl conforming elements of the second type [15, Th. 8.15].

As example for Case 2, we start from the standard lowest order Nédélec finite element space $\boldsymbol{Q}_{h}^{\mathrm{NE}} \subset \boldsymbol{Q}$, see, e.g., [15, Chap. 5] and use suitable bubble functions for an enrichment. To obtain a uniformly stable pair, it is not sufficient to guarantee $\Pi_{h}$ sym dev $\boldsymbol{Q}_{h}=\boldsymbol{B}_{h}$, and we have also to add face bubbles. Let $\mathcal{F}_{h}$ be the set of faces of the triangulation. We denote by $b_{f} \geq 0$ face bubbles satisfying

$$
\left\|\nabla b_{f}\right\|_{0} \leq C h^{-1 / 2}\left\|b_{f}\right\|_{0, f} \quad \operatorname{supp} b_{f}=\overline{T \cup T^{\prime}}, \quad f=\partial T \cap \partial T^{\prime} .
$$

In terms of these face and the element bubbles, we define $\boldsymbol{Q}_{h}=\boldsymbol{Q}_{h}^{\mathrm{NE}}+\operatorname{span}\left\{b_{T} \boldsymbol{\varepsilon}_{i}: T \in \mathcal{T}_{h}, i=1, \ldots, 5\right\}+\operatorname{span}\left\{\nabla\left(b_{f} \boldsymbol{e}_{j}\right): f \in \mathcal{F}_{h}, j=1,2,3\right\}$, where $\boldsymbol{e}_{1}, \boldsymbol{e}_{2}, \boldsymbol{e}_{3}$ is the Euclidean basis in $\mathbf{R}^{3}$.

To verify the inf-sup condition (3.6), we construct a Fortin operator $P_{h}: Q \longrightarrow Q_{h}$ satisfying

$$
\begin{array}{ll}
\left(P_{h} \boldsymbol{q}-\boldsymbol{q}, \boldsymbol{\delta}_{h}\right)_{0}=0, & \boldsymbol{q} \in \boldsymbol{Q}, \boldsymbol{\delta}_{h} \in \boldsymbol{B}_{h}, \\
\left\|P_{h} \boldsymbol{q}\right\|_{\boldsymbol{Q}} \leq C\|\boldsymbol{q}\|_{\boldsymbol{Q}}, & \boldsymbol{q} \in \boldsymbol{Q} .
\end{array}
$$

Note that $H^{1}\left(\Omega, \mathbf{R}^{3 \times 3}\right)$ is dense in $\boldsymbol{Q}$, and thus it is sufficient to construct the Fortin operator on $H^{1}\left(\Omega, \mathbf{R}^{3 \times 3}\right)$ [2, Prop. 2.8]. The construction of $P_{h}$ is based on a Helmholtz-type decomposition of $\boldsymbol{q} \in H^{1}\left(\Omega, \mathbf{R}^{3 \times 3}\right)$. Define $\boldsymbol{w} \in H_{0}^{1}\left(\Omega, \mathbf{R}^{3}\right)$ by

$$
(\nabla \boldsymbol{w}, \nabla \boldsymbol{\theta})_{0}=(\boldsymbol{q}, \nabla \boldsymbol{\theta})_{0}, \quad \boldsymbol{\theta} \in H_{0}^{1}\left(\Omega, \mathbf{R}^{3}\right),
$$

and set $\boldsymbol{\psi}=\boldsymbol{q}-\nabla \boldsymbol{w}$. By construction, this gives $\operatorname{div} \boldsymbol{\psi}=\mathbf{0},\|\nabla \boldsymbol{w}\|_{0}+\|\boldsymbol{\psi}\|_{\boldsymbol{Q}} \leq 2\|\boldsymbol{q}\|_{\boldsymbol{Q}}$. Let us assume that on $\Omega$ it holds

$$
\|\boldsymbol{q}\|_{1} \leq C(\|\boldsymbol{q}\|+\|\operatorname{curl} \boldsymbol{q}\|+\|\operatorname{div} \boldsymbol{q}\|), \quad \boldsymbol{q} \in H^{1}\left(\Omega, \mathbf{R}^{3 \times 3}\right),
$$

see, e.g., [15, Th. 3.50 and Cor. 3.51]. Then $\psi \in H^{1}\left(\Omega, \mathbf{R}^{3 \times 3}\right)$.

Now, choose a suitable approximation $\boldsymbol{w}_{h}$ in the $H^{1}$ conforming lowest order finite element space

$$
\sum_{f \in \mathcal{F}}\left\|\boldsymbol{w}-\boldsymbol{w}_{h}\right\|_{0, f}^{2} \leq C h\|\nabla \boldsymbol{w}\|_{0}^{2}
$$

[24], and choose a suitable approximation $\boldsymbol{\psi}_{h} \in \boldsymbol{Q}_{h}^{\mathrm{NE}}$ such that

$$
\left\|\boldsymbol{\psi}-\boldsymbol{\psi}_{h}\right\|_{0} \leq C h\|\boldsymbol{\psi}\|_{1} \text {. }
$$

$[22,23]$. Observing that $\nabla w_{h} \in \boldsymbol{Q}_{h}$, we define

$$
P_{h} \boldsymbol{q}=\nabla \boldsymbol{w}_{h}+\boldsymbol{\psi}_{h}-\sum_{f \in \mathcal{F}_{h}} \nabla\left(b_{f} \boldsymbol{a}_{f}\right)-\sum_{T \in \mathcal{T}_{h}} b_{T} \boldsymbol{a}_{T} \in \boldsymbol{Q}_{h}
$$

where $\boldsymbol{a}_{f}=\sum_{j=1}^{3} a_{f, j} \boldsymbol{e}_{j}$ and $\boldsymbol{a}_{T}=\sum_{i=1}^{5} a_{T, i} \varepsilon_{i}$ with coefficients given by

$$
a_{f, j}=\frac{\left(\boldsymbol{w}-\boldsymbol{w}_{h}, \boldsymbol{e}_{j}\right)_{0, f}}{\left(b_{f} \boldsymbol{e}_{j}, \boldsymbol{e}_{j}\right)_{0, f}}, \quad a_{T, i}=\frac{\left(\boldsymbol{\psi}-\boldsymbol{\psi}_{h}, \boldsymbol{\varepsilon}_{i}\right)_{0, T}}{\left(b_{T} \boldsymbol{\varepsilon}_{i}, \boldsymbol{\varepsilon}_{i}\right)_{0, T}} .
$$

Exploiting the orthogonality $\left(\varepsilon_{i}, \varepsilon_{j}\right)=0$ for $i \neq j$, we find

$$
\left(\boldsymbol{\psi}_{h}-\boldsymbol{\psi}, \boldsymbol{\varepsilon}_{i}\right)_{0, T}=a_{T, i}\left(b_{T} \boldsymbol{\varepsilon}_{i}, \boldsymbol{\varepsilon}_{i}\right)_{0, T}=\left(b_{T} \boldsymbol{a}_{T}, \boldsymbol{\varepsilon}_{i}\right)_{0, T}
$$


and using integration by parts and the fact that $\varepsilon_{i}$ is constant on $T$, we get

$$
\begin{aligned}
\left(\nabla \boldsymbol{w}_{h}-\nabla \boldsymbol{w}, \boldsymbol{\varepsilon}_{i}\right)_{0, T} & =\left(\boldsymbol{w}_{h}-\boldsymbol{w}, \boldsymbol{\varepsilon}_{i} \boldsymbol{n}_{T}\right)_{0, \partial T} \\
& =\sum_{f}\left(b_{f} \boldsymbol{a}_{f}, \boldsymbol{\varepsilon}_{i} \boldsymbol{n}_{T}\right)_{0, \partial T}=\sum_{f}\left(\nabla\left(b_{f} \boldsymbol{a}_{f}\right), \boldsymbol{\varepsilon}_{i}\right)_{0, T},
\end{aligned}
$$

where $\boldsymbol{n}_{T}$ denotes the outer normal vector on $\partial T$. To verify $(4.4 \mathrm{a})$, we know that each $\boldsymbol{\delta}_{h} \in \boldsymbol{B}_{h}$ can be written as $\boldsymbol{\delta}_{h}=\sum_{T, i} \delta_{T, i} \chi_{T} \boldsymbol{\varepsilon}_{i} \in \boldsymbol{B}_{h}$, where $\chi_{T}$ is the characteristic function on $T$. Thus it is sufficient to consider

$$
\left(P_{h} \boldsymbol{q}-\boldsymbol{q}, \boldsymbol{\varepsilon}_{i}\right)_{0, T}=\left(\nabla \boldsymbol{w}_{h}-\nabla \boldsymbol{w}-\sum_{f \in \mathcal{F}_{h}} \nabla\left(b_{f} \boldsymbol{a}_{f}\right), \boldsymbol{\varepsilon}_{i}\right)_{0, T}+\left(\boldsymbol{\psi}_{h}-\boldsymbol{\psi}-b_{T} \boldsymbol{a}_{T}, \boldsymbol{\varepsilon}_{i}\right)_{0, T}=0 .
$$

Next, we show (4.4b). Using the scaling properties of the bubble functions, the approximation properties (4.6), (4.7) and the definition of the coefficients (4.8), we get in terms of (4.5)

$$
\begin{aligned}
\left\|\sum_{T \in \mathcal{T}_{h}} b_{T} \boldsymbol{a}_{T}\right\|_{\boldsymbol{Q}}^{2} & \leq C \sum_{T \in \mathcal{T}_{h}}\left|\boldsymbol{a}_{T}\right|^{2}\left\|\nabla b_{T}\right\|_{0, T}^{2} \leq C h^{-2} \sum_{T \in \mathcal{T}_{h}}\left|\boldsymbol{a}_{T}\right|^{2}\left\|b_{T}\right\|_{0, T}^{2} \\
& \leq C h^{-2} \sum_{T \in \mathcal{T}_{h}}\left\|\boldsymbol{\psi}-\boldsymbol{\psi}_{h}\right\|_{0, T}^{2} \leq C\|\boldsymbol{\psi}\|_{1}^{2} \leq C\|\boldsymbol{\psi}\|_{\boldsymbol{Q}}^{2}, \\
\left\|\sum_{f \in \mathcal{F}_{h}} \nabla\left(b_{f} \boldsymbol{a}_{f}\right)\right\|_{0}^{2} & \leq C \sum_{f \in \mathcal{F}_{h}}\left|\boldsymbol{a}_{f}\right|^{2}\left\|\nabla b_{f}\right\|_{0}^{2} \leq C h^{-1} \sum_{f \in \mathcal{F}_{h}}\left|\boldsymbol{a}_{f}\right|^{2}\left\|b_{f}\right\|_{0, f}^{2} \\
& \leq C h^{-1} \sum_{f \in \mathcal{F}_{h}}\left\|\boldsymbol{w}-\boldsymbol{w}_{h}\right\|_{0, f}^{2} \leq C\|\nabla \boldsymbol{w}\|_{0}^{2},
\end{aligned}
$$

(where $|\cdot|$ is the Euclidean norm), which finally gives

$$
\left\|P_{h} \boldsymbol{q}\right\|_{\boldsymbol{Q}} \leq\left\|\nabla \boldsymbol{w}_{h}\right\|_{\boldsymbol{E}}+\left\|\sum_{f \in \mathcal{F}_{h}} \nabla\left(b_{f} \boldsymbol{a}_{f}\right)\right\|_{\boldsymbol{E}}+\left\|\boldsymbol{\psi}_{h}\right\|_{\boldsymbol{Q}}+\left\|\sum_{T \in \mathcal{T}_{h}} b_{T} \boldsymbol{a}_{T} \boldsymbol{\varepsilon}_{i}\right\|_{\boldsymbol{Q}} \leq C\|\boldsymbol{q}\|_{\boldsymbol{Q}}
$$

Theorem 4.4. Under the assumption that Case 1 or Case 2 hold and that the solution is smooth enough, we find

$$
\left\|\boldsymbol{u}-\boldsymbol{u}_{h}\right\|_{\boldsymbol{V}}+\left\|\boldsymbol{p}-\boldsymbol{p}_{h}\right\|_{\boldsymbol{Q}}=\mathcal{O}(h) .
$$

Moreover, in Case 2 we also find $\left\|\boldsymbol{\beta}-\boldsymbol{\beta}_{h}\right\|_{\boldsymbol{Q}^{\prime}}=\mathcal{O}(h)$.

Proof. The Case 1 follows directly from Theorem 4.1 and (4.3), and we have to consider only Case 2 in more detail. In Case 2 , we use $\left(\boldsymbol{\beta}-\boldsymbol{\beta}_{h}, \boldsymbol{q}_{h}-\boldsymbol{p}\right)_{0} \leq$ $\left\|\boldsymbol{\beta}-\boldsymbol{\beta}_{h}\right\|_{\boldsymbol{Q}^{\prime}}\left\|\boldsymbol{p}-\boldsymbol{q}_{h}\right\|_{\boldsymbol{Q}}$. In a next step, we bound the error in $\left\|\boldsymbol{\beta}-\boldsymbol{\beta}_{h}\right\|_{\boldsymbol{Q}^{\prime}}$ by the primal error and a best approximation result yielding in terms of (3.6)

$$
\begin{aligned}
\left\|\boldsymbol{\beta}-\boldsymbol{\beta}_{h}\right\|_{\boldsymbol{Q}^{\prime}} & \leq\left\|\boldsymbol{\beta}-\Pi_{h} \boldsymbol{\beta}\right\|_{\boldsymbol{Q}^{\prime}}+c_{\mathrm{stab}}^{-1} \sup _{\boldsymbol{q}_{h} \in \boldsymbol{Q}_{h}, \boldsymbol{q}_{h} \neq 0} \frac{\left(\Pi_{h} \boldsymbol{\beta}-\boldsymbol{\beta}_{h}, \boldsymbol{q}_{h}\right)_{0}}{\left\|\boldsymbol{q}_{h}\right\|_{\boldsymbol{Q}}} \\
& \leq\left(1+c_{\mathrm{stab}}^{-1}\right)\left\|\boldsymbol{\beta}-\Pi_{h} \boldsymbol{\beta}\right\|_{\boldsymbol{Q}^{\prime}}+c_{\mathrm{stab}}^{-1} \sup _{\boldsymbol{q}_{h} \in \boldsymbol{Q}_{h}, \boldsymbol{q}_{h} \neq 0} \frac{\left(\boldsymbol{\beta}-\boldsymbol{\beta}_{h}, \boldsymbol{q}_{h}\right)_{0}}{\left\|\boldsymbol{q}_{h}\right\|_{\boldsymbol{Q}}} \\
& \leq \mathcal{O}(h)+c_{\text {stab }}^{-1}\left\|\boldsymbol{p}-\boldsymbol{p}_{h}\right\|_{\boldsymbol{Q}} .
\end{aligned}
$$

5. A generalized Newton method for the mixed finite element approximation. In the primal-dual formulation the dissipation inequality (3.3c) is a local equation which can be directly evaluated with a standard return mapping algorithm, i.e., we have a nonlinear response function for the plastic strain depending on the trial stress. This observation can be used to define an efficient nonlinear iterative solver.

As it is well known, the classical radial return algorithm can be interpreted within the abstract framework of generalized Newton methods. More precisely, we use a semi-smooth Newton method to solve numerically the variational inequality. Local variational inequalities can be easily rewritten as non-linear equality constraints in 
terms of NCP-functions. We refer to [4] for a general construction and analysis and to [10] for some stabilization techniques.

We generalize the classical radial return algorithm for local plasticity to our nonlocal situation, see, e.g., [25, Chap. 3.3.1]. Introducing the deviatoric trial stress $\boldsymbol{\theta}_{h}=2 \mu \Pi_{h} \operatorname{dev} \boldsymbol{\varepsilon}\left(\boldsymbol{u}_{h}\right)-\boldsymbol{\beta}_{h}$, we define locally on each cell

$$
R_{h}\left(\boldsymbol{\theta}_{h}\right)=\alpha_{h} \boldsymbol{\theta}_{h}, \quad \alpha_{h}=\frac{\max \left\{0,\left|\boldsymbol{\theta}_{h}\right|-K_{0}\right\}}{2 \mu\left|\boldsymbol{\theta}_{h}\right|} .
$$

Lemma 5.1. Let $\left(\boldsymbol{u}_{h}, \boldsymbol{\beta}_{h}\right) \in \boldsymbol{V}_{h} \times \boldsymbol{B}_{h}$ be given. Then, the discrete dissipation inequality

$$
j\left(\boldsymbol{\eta}_{h}\right) \geq j\left(\varepsilon_{h}^{p}\right)+\left(\operatorname{dev} \boldsymbol{\sigma}_{h}-\boldsymbol{\beta}_{h}, \boldsymbol{\eta}_{h}-\varepsilon_{h}^{p}\right)_{0}, \quad \boldsymbol{\eta}_{h} \in \boldsymbol{E}_{h} .
$$

is satisfied for the plastic strain $\varepsilon_{h}^{p}=R_{h}\left(\boldsymbol{\theta}_{h}\right)$ and the stress $\boldsymbol{\sigma}_{h}=\mathbb{C}:\left(\varepsilon\left(\boldsymbol{u}_{h}\right)-\varepsilon_{h}^{p}\right)$.

Proof. For convenience of the reader, we recall the basic steps of the proof. We find $\left|\boldsymbol{\theta}_{h}\right|-\max \left\{0,\left|\boldsymbol{\theta}_{h}\right|-K_{0}\right\} \leq K_{0}$ and thus

$$
\left(\boldsymbol{\theta}_{h}-2 \mu R_{h}\left(\boldsymbol{\theta}_{h}\right)\right): \boldsymbol{\eta}_{h} \leq K_{0}\left|\boldsymbol{\eta}_{h}\right|, \quad \boldsymbol{\eta}_{h} \in \boldsymbol{E}_{h} .
$$

Then, the identity $K_{0}\left|R_{h}\left(\boldsymbol{\theta}_{h}\right)\right|-\left(\boldsymbol{\theta}_{h}-2 \mu R_{h}\left(\boldsymbol{\theta}_{h}\right)\right): R_{h}\left(\boldsymbol{\theta}_{h}\right)=0$ and $\operatorname{dev} \varepsilon_{h}^{p}=\varepsilon_{h}^{p}$ yields

$$
\begin{aligned}
K_{0}\left|\boldsymbol{\eta}_{h}\right| & \geq K_{0}\left|R_{h}\left(\boldsymbol{\theta}_{h}\right)\right|+\left(\boldsymbol{\theta}_{h}-2 \mu R_{h}\left(\boldsymbol{\theta}_{h}\right)\right):\left(\boldsymbol{\eta}_{h}-R_{h}\left(\boldsymbol{\theta}_{h}\right)\right) \\
& =K_{0}\left|\varepsilon_{h}^{p}\right|+\left(\operatorname{dev} \boldsymbol{\sigma}_{h}-\boldsymbol{\beta}_{h}\right):\left(\boldsymbol{\eta}_{h}-\boldsymbol{\varepsilon}_{h}^{p}\right), \quad \boldsymbol{\eta}_{h} \in \boldsymbol{E}_{h} .
\end{aligned}
$$

Inserting the radial return in (3.3c) rewrites (3.3) as a nonlinear variational equality: find $\left(\boldsymbol{u}_{h}, \boldsymbol{p}_{h}, \boldsymbol{\beta}_{h}\right) \in \boldsymbol{V}_{h} \times \boldsymbol{Q}_{h} \times \boldsymbol{B}_{h}$ such that

$$
\begin{array}{ll}
\left(\mathbb{C}: \boldsymbol{\varepsilon}\left(\boldsymbol{u}_{h}\right)-2 \mu R_{h}\left(2 \mu \operatorname{dev} \boldsymbol{\varepsilon}\left(\boldsymbol{u}_{h}\right)-\boldsymbol{\beta}_{h}\right), \boldsymbol{\varepsilon}\left(\boldsymbol{v}_{h}\right)\right)_{0}=\ell\left(\boldsymbol{v}_{h}\right), & \boldsymbol{v}_{h} \in \boldsymbol{V}_{h} \\
\left(\boldsymbol{\beta}_{h}, \boldsymbol{q}_{h}\right)_{0}=\left(\boldsymbol{p}_{h}, \boldsymbol{q}_{h}\right)_{\boldsymbol{Q}}, & \boldsymbol{q}_{h} \in \boldsymbol{Q}_{h} \\
\left(R_{h}\left(2 \mu \operatorname{dev} \boldsymbol{\varepsilon}\left(\boldsymbol{u}_{h}\right)-\boldsymbol{\beta}_{h}\right), \boldsymbol{\delta}_{h}\right)_{0}=\left(\boldsymbol{p}_{h}, \boldsymbol{\delta}_{h}\right)_{0}, & \boldsymbol{\delta}_{h} \in \boldsymbol{B}_{h}
\end{array}
$$

This defines $\varepsilon_{h}^{p}=R_{h}\left(2 \mu \operatorname{dev} \varepsilon\left(\boldsymbol{u}_{h}\right)-\boldsymbol{\beta}_{h}\right)=\Pi_{h} \operatorname{sym} \operatorname{dev} \boldsymbol{p}_{h}$.

For a consistent linearization, we select a realization $\mathbb{R}_{h}\left(\boldsymbol{\theta}_{h}\right) \in \partial R_{h}\left(\boldsymbol{\theta}_{h}\right)$. For $\left|\boldsymbol{\theta}_{h}\right| \neq 0$ the sub-differential is given by

$$
\partial R_{h}\left(\boldsymbol{\theta}_{h}\right)=\frac{\partial\left(\max \left\{0,\left|\boldsymbol{\theta}_{h}\right|-K_{0}\right\}\right)}{2 \mu} \frac{\boldsymbol{\theta}_{h}}{\left|\boldsymbol{\theta}_{h}\right|} \otimes \frac{\boldsymbol{\theta}_{h}}{\left|\boldsymbol{\theta}_{h}\right|}+\alpha_{h}\left(\mathrm{id}-\frac{\boldsymbol{\theta}_{h}}{\left|\boldsymbol{\theta}_{h}\right|} \otimes \frac{\boldsymbol{\theta}_{h}}{\left|\boldsymbol{\theta}_{h}\right|}\right) .
$$

The mixed system (5.1) is solved with a generalized Newton method (see Algorithm 1). The method is well defined since the Newton linearization is always regular.

Lemma 5.2. A unique solution of the linearized problem in step S2) of Algorithm 1 exists.

Proof. We have to show that the equation $B_{h}^{k}\left[\left(\boldsymbol{v}_{h}, \boldsymbol{q}_{h}, \boldsymbol{\delta}_{h}\right),(\cdot, \cdot, \cdot)\right]=0$ only has the trivial solution. For all $\boldsymbol{r}_{h} \in \boldsymbol{Q}_{h}$ holds

$$
0=B_{h}^{k}\left[\left(\boldsymbol{v}_{h}, \boldsymbol{q}_{h}, \boldsymbol{\delta}_{h}\right),\left(\mathbf{0}, \boldsymbol{r}_{h}, \mathbf{0}\right)\right]=\left(\boldsymbol{q}_{h}, \boldsymbol{r}_{h}\right)_{\boldsymbol{Q}}-\left(\boldsymbol{\delta}_{h}, \boldsymbol{r}_{h}\right)_{0} .
$$

Next, we observe on all elements where $\gamma_{h}^{k}=\max \left\{0,\left|\boldsymbol{\theta}_{h}^{k}\right|-K_{0}\right\}>0$

$$
\begin{aligned}
\boldsymbol{\delta}_{h}: \mathbb{R}_{h}^{k}: \boldsymbol{\delta}_{h} & =\frac{1}{2 \mu} \frac{\left(\boldsymbol{\theta}_{h}^{k}: \boldsymbol{\delta}_{h}\right)^{2}}{\left|\boldsymbol{\theta}_{h}^{k}\right|^{2}}+\frac{\gamma_{h}^{k}}{2 \mu\left|\boldsymbol{\theta}_{h}^{k}\right|}\left(\boldsymbol{\delta}_{h}: \boldsymbol{\delta}_{h}-\frac{\left(\boldsymbol{\theta}_{h}^{k}: \boldsymbol{\delta}_{h}\right)^{2}}{\left|\boldsymbol{\theta}_{h}^{k}\right|^{2}}\right) \\
& =\frac{\gamma_{h}^{k}}{2 \mu\left|\boldsymbol{\theta}_{h}^{k}\right|} \boldsymbol{\delta}_{h}: \boldsymbol{\delta}_{h}+\frac{K_{0}}{2 \mu} \frac{\left(\boldsymbol{\theta}_{h}^{k}: \boldsymbol{\delta}_{h}\right)^{2}}{\left|\boldsymbol{\theta}_{h}^{k}\right|^{2}} \geq 0
\end{aligned}
$$


S0) Choose start iterates $\left(\boldsymbol{u}_{h}^{0}, \boldsymbol{p}_{h}^{0}, \boldsymbol{\beta}_{h}^{0}\right) \in \boldsymbol{V}_{h} \times \boldsymbol{Q}_{h} \times \boldsymbol{B}_{h}$. Set $k=0$.

S1) Compute the deviatoric trial stress $\boldsymbol{\theta}^{k}=2 \mu \operatorname{dev} \varepsilon\left(\boldsymbol{u}_{h}^{k}\right)-\boldsymbol{\beta}_{h}^{k}$ and the residual

$$
\begin{aligned}
F_{h}^{k}\left[\boldsymbol{v}_{h}, \boldsymbol{q}_{h}, \boldsymbol{\delta}_{h}\right]=(\mathbb{C}: & \left.\boldsymbol{\varepsilon}\left(\boldsymbol{u}_{h}^{k}\right)_{0}-2 \mu R_{h}\left(\boldsymbol{\theta}^{k}\right), \boldsymbol{\varepsilon}\left(\boldsymbol{v}_{h}\right)\right)_{0}-\ell\left(\boldsymbol{v}_{h}\right) \\
& +\left(\boldsymbol{p}_{h}^{k}, \boldsymbol{q}_{h}\right)_{\boldsymbol{Q}}-\left(\boldsymbol{\beta}_{h}^{k}, \boldsymbol{q}_{h}\right)_{0} \\
& +\left(R_{h}\left(\boldsymbol{\theta}^{k}\right)-\boldsymbol{p}_{h}^{k}, \boldsymbol{\delta}_{h}\right)_{0} .
\end{aligned}
$$

If the residual norm $\rho_{k}=\left\|F_{h}^{k}\right\|$ is small enough, STOP.

S2) Compute the solution $\left(\Delta \boldsymbol{u}_{h}^{k}, \Delta \boldsymbol{p}_{h}^{k}, \Delta \boldsymbol{\beta}_{h}^{k}\right) \in \boldsymbol{V}_{h} \times \boldsymbol{Q}_{h} \times \boldsymbol{B}_{h}$ of the linear problem

$$
B_{h}^{k}\left[\left(\Delta \boldsymbol{u}_{h}^{k}, \Delta \boldsymbol{p}_{h}^{k}, \Delta \boldsymbol{\beta}_{h}^{k}\right),\left(\boldsymbol{v}_{h}, \boldsymbol{q}_{h}, \boldsymbol{\delta}_{h}\right)\right]=-F_{h}^{k}\left[\boldsymbol{v}_{h}, \boldsymbol{q}_{h}, \boldsymbol{\delta}_{h}\right]
$$

for $\left(\boldsymbol{v}_{h}, \boldsymbol{q}_{h}, \boldsymbol{\delta}_{h}\right) \in \boldsymbol{V}_{h} \times \boldsymbol{Q}_{h} \times \boldsymbol{B}_{h}$, where the symmetric bilinear form is given by

$$
\begin{aligned}
B_{h}^{k}\left[\left(\Delta \boldsymbol{u}_{h}, \Delta \boldsymbol{p}_{h}, \Delta \boldsymbol{\beta}_{h}\right),\left(\boldsymbol{v}_{h}, \boldsymbol{q}_{h}, \boldsymbol{\delta}_{h}\right)\right]=\left(\mathbb{C}: \varepsilon\left(\Delta \boldsymbol{u}_{h}\right), \boldsymbol{\varepsilon}\left(\boldsymbol{v}_{h}\right)\right)_{0} \\
-4 \mu^{2}\left(\mathbb{R}_{h}^{k}: \operatorname{dev} \boldsymbol{\varepsilon}\left(\Delta \boldsymbol{u}_{h}\right), \operatorname{dev} \boldsymbol{\varepsilon}\left(\boldsymbol{v}_{h}\right)\right)_{0}+2 \mu\left(\mathbb{R}_{h}^{k}: \Delta \boldsymbol{\beta}_{h}, \operatorname{dev} \boldsymbol{\varepsilon}\left(\boldsymbol{v}_{h}\right)\right)_{0} \\
+\left(\Delta \boldsymbol{p}_{h}, \boldsymbol{q}_{h}\right)_{\boldsymbol{Q}}-\left(\Delta \boldsymbol{\beta}_{h}, \boldsymbol{q}_{h}\right)_{0} \\
+2 \mu\left(\mathbb{R}_{h}^{k}: \operatorname{dev} \boldsymbol{\varepsilon}\left(\Delta \boldsymbol{u}_{h}\right), \boldsymbol{\delta}_{h}\right)_{0}-\left(\Delta \boldsymbol{p}_{h}, \boldsymbol{\delta}_{h}\right)_{0}-\left(\mathbb{R}_{h}^{k}: \Delta \boldsymbol{\beta}_{h}, \boldsymbol{\delta}_{h}\right)_{0}
\end{aligned}
$$

with $\mathbb{R}_{h}^{k}=\frac{1}{2 \mu} \frac{\boldsymbol{\theta}_{h}^{k}}{\left|\boldsymbol{\theta}_{h}^{k}\right|} \otimes \frac{\boldsymbol{\theta}_{h}^{k}}{\left|\boldsymbol{\theta}_{h}^{k}\right|}+\alpha_{h}\left(\mathrm{id}-\frac{\boldsymbol{\theta}_{h}^{k}}{\left|\boldsymbol{\theta}_{h}^{k}\right|} \otimes \frac{\boldsymbol{\theta}_{h}^{k}}{\left|\boldsymbol{\theta}_{h}^{k}\right|}\right)$ if $\alpha_{h}=\frac{\max \left\{0,\left|\boldsymbol{\theta}_{h}^{k}\right|-K_{0}\right\}}{2 \mu\left|\boldsymbol{\theta}_{h}^{k}\right|}>0$ and $\mathbb{R}_{h}^{k}=0$ else;

S3) Choose a suitable damping parameter $s^{k} \in(0,1]$ and set

$$
\left(\boldsymbol{u}_{h}^{k+1}, \boldsymbol{p}_{h}^{k+1}, \boldsymbol{\beta}_{h}^{k+1}\right)=\left(\boldsymbol{u}_{h}^{k}, \boldsymbol{p}_{h}^{k}, \boldsymbol{\beta}_{h}^{k}\right)+s^{k}\left(\Delta \boldsymbol{u}_{h}^{k}, \Delta \boldsymbol{p}_{h}^{k}, \Delta \boldsymbol{\beta}_{h}^{k}\right) .
$$

Set $k:=k+1$ and go to S1).

Algorithm 1: Generalized Newton algorithm for the solution of the primal-dual system (5.1).

and

$$
\begin{aligned}
4 \mu^{2} \operatorname{dev} & \boldsymbol{\varepsilon}\left(\boldsymbol{v}_{h}\right): \mathbb{R}_{h}^{k}: \operatorname{dev} \boldsymbol{\varepsilon}\left(\boldsymbol{v}_{h}\right) \\
& =2 \mu \frac{\left(\boldsymbol{\theta}_{h}^{k}: \boldsymbol{\varepsilon}\left(\boldsymbol{v}_{h}\right)\right)^{2}}{\left|\boldsymbol{\theta}_{h}^{k}\right|^{2}}+\frac{2 \mu \gamma_{h}^{k}}{\left|\boldsymbol{\theta}_{h}^{k}\right|}\left(\left|\operatorname{dev} \varepsilon\left(\boldsymbol{v}_{h}\right)\right|^{2}-\frac{\left(\boldsymbol{\theta}_{h}^{k}: \boldsymbol{\varepsilon}\left(\boldsymbol{v}_{h}\right)\right)^{2}}{\left|\boldsymbol{\theta}_{h}^{k}\right|^{2}}\right) \\
& \leq 2 \mu \frac{\left(\boldsymbol{\theta}_{h}^{k}: \boldsymbol{\varepsilon}\left(\boldsymbol{v}_{h}\right)\right)^{2}}{\left|\boldsymbol{\theta}_{h}^{k}\right|^{2}}+2 \mu\left(\left|\varepsilon\left(\boldsymbol{v}_{h}\right)\right|^{2}-\frac{\left(\boldsymbol{\theta}_{h}^{k}: \boldsymbol{\varepsilon}\left(\boldsymbol{v}_{h}\right)\right)^{2}}{\left|\boldsymbol{\theta}_{h}^{k}\right|^{2}}\right) \\
& \leq \boldsymbol{\varepsilon}\left(\boldsymbol{v}_{h}\right): \mathbb{C}: \boldsymbol{\varepsilon}\left(\boldsymbol{v}_{h}\right) .
\end{aligned}
$$

Then,

$$
\begin{aligned}
0 & =B_{h}^{k}\left[\left(\boldsymbol{v}_{h}, \boldsymbol{q}_{h}, \boldsymbol{\delta}_{h}\right),\left(\boldsymbol{v}_{h}, \boldsymbol{q}_{h},-\boldsymbol{\delta}_{h}\right)\right] \\
= & \left(\mathbb{C}: \varepsilon\left(\boldsymbol{v}_{h}\right), \boldsymbol{\varepsilon}\left(\boldsymbol{v}_{h}\right)\right)_{0}-4 \mu^{2}\left(\mathbb{R}_{h}^{k}: \operatorname{dev} \varepsilon\left(\boldsymbol{v}_{h}\right), \operatorname{dev} \boldsymbol{\varepsilon}\left(\boldsymbol{v}_{h}\right)\right)_{0} \\
& \quad+\left(\boldsymbol{q}_{h}, \boldsymbol{q}_{h}\right)_{\boldsymbol{Q}}+\left(\mathbb{R}_{h}^{k}: \boldsymbol{\delta}_{h}, \boldsymbol{\delta}_{h}\right)_{0} \\
\geq & \left(\boldsymbol{q}_{h}, \boldsymbol{q}_{h}\right)_{\boldsymbol{Q}}
\end{aligned}
$$

gives $\boldsymbol{q}_{h}=\mathbf{0}$, and (5.2) yields $\boldsymbol{\delta}_{h}=\mathbf{0}$ due to our assumption $\boldsymbol{B}_{h}^{0}=\boldsymbol{B}_{h}$. Now, testing 
with $\left(\mathbf{0}, \mathbf{0}, \operatorname{dev} \varepsilon\left(\boldsymbol{v}_{h}\right)\right)$ gives $\operatorname{dev} \boldsymbol{\varepsilon}\left(\boldsymbol{v}_{h}\right)=\mathbf{0}$ and finally testing with $\left(\boldsymbol{v}_{h}, \mathbf{0}, \mathbf{0}\right)$ yields $\boldsymbol{v}_{h}=\mathbf{0}$.

The Lemma guarantees that our solver is well-defined and-since the nonlinearity in (5.1) is strongly semi-smooth-local super-linear convergence is guaranteed.

REMARK 5.3. We note that there exists always a solution $\left(\Delta \boldsymbol{u}_{h}^{k}, \Delta \boldsymbol{p}_{h}^{k}, \Delta \boldsymbol{\beta}_{h}^{k}\right)$ in S2) assuming that our start iterate satisfies $\left(\boldsymbol{\beta}_{h}^{0}, \boldsymbol{q}_{h}\right)_{0}=\left(\boldsymbol{p}_{h}^{0}, \boldsymbol{q}_{h}\right)_{\boldsymbol{Q}}$ for all $\boldsymbol{q}_{h} \in \boldsymbol{Q}_{h}$.

6. Numerical example. We apply our solver for a test configuration defined in [18], cf. Fig. 6.1. In our example we use the Lamé parameters $\mu=80193.80$ $\left[\mathrm{N} / \mathrm{mm}^{2}\right], \lambda=110743.82\left[\mathrm{~N} / \mathrm{mm}^{2}\right]$, the yield stress $K_{0}=367.42\left[\mathrm{~N} / \mathrm{mm}^{2}\right]$, a very small hardening modulus $H_{0}=0.0001 \mu$ and the length scale parameter $L_{0}=0.1$. The bottom of the configuration is fixed (homogeneous Dirichlet boundary conditions), on the top a traction load of $70\left[\mathrm{~N} / \mathrm{mm}^{2}\right]$ is applied.

The computation is realized in the parallel finite element code $\mathrm{M}++[29]$. For simplicity, we use standard finite element spaces on hexahedral meshes: trilinear elements $\boldsymbol{V}_{h}$ for the displacements, lowest order hexahedral Nédélec elements $\boldsymbol{Q}_{h}$ for the plastic variable, and element-wise constant tensors $\boldsymbol{B}_{h}$ for the back stress. In contrast to our theoretical results, we do not enrich our space. In our example we observe that the bilinear form $B_{h}[\cdot, \cdot]$ is regular which holds if and only if $\boldsymbol{B}_{h}^{0} \neq \boldsymbol{B}_{h}$.

The linearized problems are solved with the BiCGStab method. As preconditioner, we apply a fully parallel multigrid method using overlapping block Gauß-Seidel smoothing and exact coarse problem solver, cf. [30] for details on the parallel data structure and the solution method. In order to obtain a robust method, very large blocks in the smoother and multiple smoothing is used, so that in all steps and on all levels less than 10 BiCGStab iterations are sufficient to reduce the residual norm by the factor $10^{-6}$. The semi-smooth Newton method show super-linear convergence (see Tab. 6.1). However, our numerical results indicate a linear dependency on the refinement level.
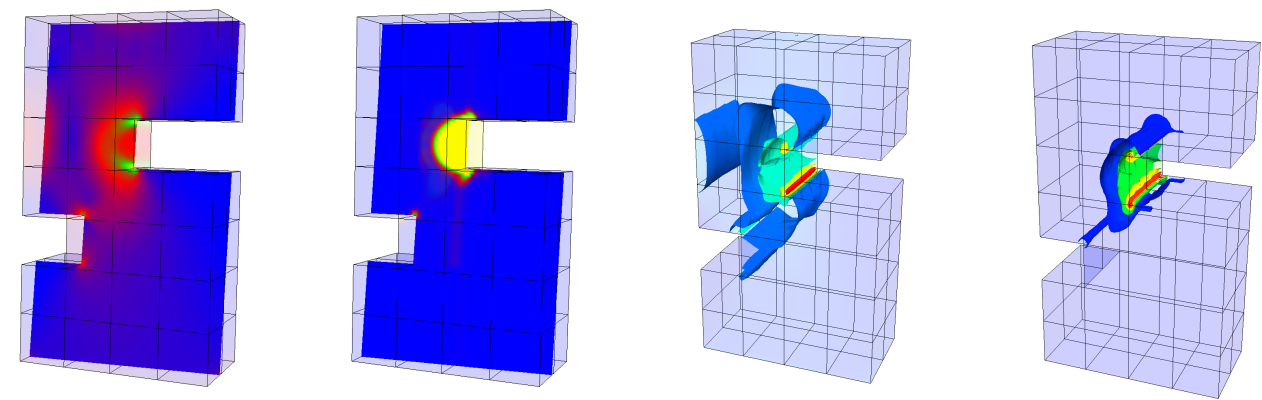

FIG. 6.1. Final norm distribution of the stress $\boldsymbol{\sigma}_{h}$ and the plastic variable $\boldsymbol{p}_{h}$. On the left a $2 D$ cut is shown whereas on the right the $3 D$ solution is visualized.

The numerical results in Tab. 6.2 show the convergence of the computed values with respect to the refinement level. The plastic region (i.e., all cells where $\varepsilon_{h}^{p}$ is not zero) is identified correctly already on very coarse meshes, but a precise computation of displacements requires the finest level; by extrapolation of the computed data an accuracy of approx. $5 \%$ can be estimated. Due to the reentrant corners of the computational domain we do not expect full regularity and thus no convergence of $\sigma_{h}$ in $L_{\infty}$. The resulting distribution of the stress and the plastic variable on level 4 are illustrated in Fig. 6.1 (the background mesh is plotted on level 0). 


\begin{tabular}{r|r|r|r|c|c|c|c|c|c|c}
$j$ & \multicolumn{1}{|c|}{$\rho_{0}$} & \multicolumn{1}{c|}{$\rho_{1}$} & \multicolumn{1}{|c|}{$\rho_{2}$} & $\rho_{3}$ & $\rho_{4}$ & $\rho_{5}$ & $\rho_{6}$ & $\rho_{7}$ & $\rho_{8}$ & $\rho_{9}$ \\
\hline 0 & 160.3 & 16.4 & 0.06 & 0.000001 & $\varepsilon$ & & & & & \\
1 & 89.6 & 46.2 & 17.24 & 0.970748 & 0.0024 & $\varepsilon$ & & & & \\
2 & 47.1 & 39.5 & 13.11 & 2.842823 & 0.0726 & 0.000023 & $\varepsilon$ & & & \\
3 & 24.1 & 12.4 & 11.68 & 8.649902 & 7.7275 & 1.572126 & 0.190 & 0.0011 & $\varepsilon$ & \\
4 & 12.2 & 6.7 & 6.13 & 4.661071 & 4.5857 & 1.235850 & 0.156 & 0.0016 & 0.000002 & $\varepsilon$
\end{tabular}

TABLE 6.1

Convergence history (up to $\varepsilon \leq 10^{-9}$ ) of the semi-smooth Newton method. For the first two steps on level $j=4$ a line search was required in order to reduce the residual.

\begin{tabular}{r|r|r|c|c|r}
$\mathrm{j}$ & \multicolumn{1}{c|}{ d.o.f. } & \# cells & \# plastic cells & $\left|\boldsymbol{u}^{h}(\boldsymbol{z})\right|$ & $\left\|\boldsymbol{\sigma}^{h}\right\|_{\infty}$ \\
\hline 0 & 1426 & 50 & 8 & 0.0167 & 581.64 \\
1 & 8903 & 400 & 77 & 0.0158 & 879.08 \\
2 & 62419 & 3200 & 836 & 0.0192 & 1161.38 \\
3 & 466499 & 25600 & 7122 & 0.0214 & 1506.84 \\
4 & 3605251 & 204800 & 60622 & 0.0228 & 1941.87
\end{tabular}

TABLE 6.2

Convergence for successive uniform refinements of the displacement at a test point $\boldsymbol{z}=(0,0,7)$ and for the stress maximum. In every refinement step each cell is divided into 8 cells.

\section{REFERENCES}

[1] J. Alberty, C. Carstensen, and D. Zarrabi, Adaptive numerical analysis in primal elastoplasticity with hardening, IMA Journal of Numerical Analysis, 171 (1999), pp. 175-204.

[2] F. Brezzi and M. Fortin, Mixed and Hybrid Finite Element Methods, Springer-Verlag Berlin, 1991.

[3] C. Carstensen, Numerical analysis of the primal problem of elastoplasticity with hardening, Numerische Mathematik, 82 (1999), pp. 577-597.

[4] P. W. Christensen, A nonsmooth Newton method for elastoplastic problems, Comput. Meth. Appl. Mech. Engrg., 191 (2002), pp. 1189-2119.

[5] J. Djoko, F. Ebobisse, A. McBride, and B. Reddy, A discontinuous Galerkin formulation for classical and gradient plasticity. Part 1: Formulation and analysis, Comp. Meth. Appl. Mech. Engrg., 196 (2007), pp. 3881-3897.

[6] - A discontinuous Galerkin formulation for classical and gradient plasticity. Part 2: Algorithms and numerical analysis, Comp. Meth. Appl. Mech. Engrg., 197 (2007), pp. 121.

[7] M. GuRTIN, On the plasticity of single crystals: free energy, microforces, plastic-strain gradients, J. Mech. Phys. Solids, 48 (2000), pp. 989-1036.

[8] M. Gurtin And L. Anand, A theory of strain-gradient plasticity for isotropic, plastically irrotational materials. Part I: Small deformations., J. Mech. Phys. Solids, 53 (2005), pp. 16241649.

[9] M. Gurtin and A. Needleman, Boundary conditions in small-deformation, single crystal plasticity that account for the Burgers vector., J. Mech. Phys. Solids, 53 (2005), pp. 1-31.

[10] C. Hager and B. Wohlmuth, Nonlinear complementarity functions for plasticity problems with frictional contact, Comput. Meth. Appl. Mech. Engrg., 198 (2009), pp. 3411-3427.

[11] W. Han and B. Reddy, Plasticity: Mathematical Theory and Numerical Analysis, SpringerVerlag Berlin, 1999.

[12] R. Hiptmair, Finite elements in computational electromagnetism, Acta Numerica, 7 (2002), pp. 237-339.

[13] A. Menzel and P. Steinmann, On the continuum formulation of higher gradient plasticity for single and polycrystals, J. Mech. Phys. Solids, 48 (2000), pp. 1777-1796.

[14] A. Mielke And S. MülleR, Lower semi-continuity and existence of minimizers in incremental 
finite-strain elastoplasticity, Z. Angew. Math. u. Mech., 86 (2006), pp. 233-250.

[15] P. Monk, Finite element methods for Maxwell's equations, Clarendon Press, Oxford, 2003.

[16] J. NédÉlec, Mixed finite elements in $\mathbb{R}^{3}$, Numer. Math., 35 (1980), pp. 315-341.

[17] P. NefF, K. Cheemiński, and H.-D. Alber, Notes on strain gradient plasticity: finite strain covariant modelling and global existence in the infinitesimal rate-independent case., Math. Models Methods Appl. Sci., 19 (2009), pp. 307-346.

[18] P. NefF, A. Sydow, and C. Wieners, Numerical approximation of incremental infinitesimal gradient plasticity, Int. J. Numer. Meth. Eng., 77 (2009), pp. 414-436.

[19] J. Ostien and K. Garikipati, A discontinuous Galerkin method for an incompatibility-based strain gradient plasticity theory. Reddy, D. (ed.), IUTAM Symposium on Theoretical, Modelling and Computational Aspects of Inelastic Media, Berlin: Springer, 2008.

[20] B. Reddy, F. Ebobisse, And A. McBride, Well-posedness of a model of strain gradient plasticity for plastically irrotational materials, Int. J. Plasticity, 24 (2008), pp. 55-73.

[21] B. D. RedDy, Mixed variational inequalities arising in elastoplasticity, Nonlinear Anal., 19 (1992), pp. 1071-1089.

[22] J. SchöBERL, Commuting quasi-interpolation operators for mixed finite elements, 2001. Texas A\&M University, Report ISC-01-10-MATH.

[23] _ - A posteriori error estimates for Maxwell equations, Math. Comp., 77 (2008), pp. 633649.

[24] R. Sсотt AND ZhaNG, Finite element interpolation of nonsmooth functions satisfying boundary conditions, Math. Comp., 54 (1990), pp. 483-493.

[25] J. C. Simo and T. J. R. Hughes, Computational Inelasticity, Springer-Verlag Berlin, 1998.

[26] B. Svendsen, Continuum thermodynamic models for crystal plasticity including the effects of geometrically necessary dislocations, J. Mech. Phys. Solids, 50 (2002), pp. 1297-1329.

[27] - On thermodynamic- and variational based formulations for inelastic continua with internal lengthscales, Comp. Meth. Appl. Mech. Eng., 48-51 (2004), pp. 5429-5452.

[28] R. Temam, Mathematical Problems in Plasticity, Bordas, Paris, 1985.

[29] C. Wieners, Distributed point objects. A new concept for parallel finite elements, in Domain Decomposition Methods in Science and Engineering, R. Kornhuber, R. Hoppe, J. Périaux, O. Pironneau, O. Widlund, and J. Xu, eds., vol. 40 of Lecture Notes in Computational Science and Engineering, Springer-Verlag Berlin, 2004, pp. 175-183.

[30] — - A geometric data structure for parallel finite elements and the application to multigrid methods with block smoothing, Computing and Visulization in Science, (2010). to appear. 


\section{IWRMM-Preprints seit 2008}

Nr. 08/01 Patrizio Neff, Antje Sydow, Christian Wieners: Numerical approximation of incremental infinitesimal gradient plasticity

Nr. 08/02 Götz Alefeld, Zhengyu Wang: Error Estimation for Nonlinear Complementarity Problems via Linear Systems with Interval Data

Nr. 08/03 Ulrich Kulisch : Complete Interval Arithmetic and its Implementation on the Computer

Nr. 08/04 Armin Lechleiter, Andreas Rieder: Newton Regularizations for Impedance Tomography: Convergence by Local Injectivity

Nr. 08/05 Vu Hoang, Michael Plum, Christian Wieners: A computer-assisted proof for photonic band gaps

Nr. 08/06 Vincent Heuveline, Peter Wittwer: Adaptive boundary conditions for exterior stationary flows in three dimensions

Nr. 08/07 Jan Mayer: Parallel Algorithms for Solving Linear Systems with Sparse Triangular Matrices

Nr. 08/08 Ulrich Kulisch : Begegnungen eines Mathematikers mit Informatik

Nr. 08/09 Tomas Dohnal, Michael Plum, Wolfgang Reichel: Localized Modes of the Linear Periodic Schrödinger Operator with a Nonlocal Perturbation

Nr. 08/10 Götz Alefeld: Verified Numerical Computation for Nonlinear Equations

Nr. 08/11 Götz Alefeld, Zhengyu Wang: Error bounds for complementarity problems with tridiagonal nonlinear functions

Nr. 08/12 Tomas Dohnal, Hannes Uecker: Coupled Mode Equations and Gap Solitons for the 2D Gross-Pitaevskii equation with a non-separable periodic potential

Nr. 09/01 Armin Lechleiter, Andreas Rieder: Towards A General Convergence Theory For Inexact Newton Regularizations

Nr. 09/02 Christian Wieners: A geometric data structure for parallel finite elements and the application to multigrid methods with block smoothing

Nr. 09/03 Arne Schneck: Constrained Hardy Space Approximation

Nr. 09/04 Arne Schneck: Constrained Hardy Space Approximation II: Numerics

Nr. 10/01 Ulrich Kulisch, Van Snyder : The Exact Dot Product As Basic Tool For Long Interval Arithmetic

Nr. 10/02 Tobias Jahnke : An Adaptive Wavelet Method for The Chemical Master Equation

Nr. 10/03 Christof Schütte, Tobias Jahnke : Towards Effective Dynamics in Complex Systems by Markov Kernel Approximation

Nr. 10/04 Tobias Jahnke, Tudor Udrescu : Solving chemical master equations by adaptive wavelet compression

Nr. 10/05 Christian Wieners, Barbara Wohlmuth : A Primal-Dual Finite Element Approximation For A Nonlocal Model in Plasticity

Eine aktuelle Liste aller IWRMM-Preprints finden Sie auf: 


\section{Kontakt}

Karlsruher Institut $f$ r Technologie (KIT) Institut $f \mathrm{r}$ Wissenschaftliches Rechnen und Mathematische Modellbildung

Prof. Dr. Christian Wieners Gesch ftsf hrender Direktor

Campus S d

Engesserstr. 6

76131 Karlsruhe

E-Mail: i rmm-sekretariat@math.uka.de

.math.kit.edu/i rmm/

\section{Herausgeber}

Karlsruher Institut f r Technologie (KIT)

Kaiserstra e 1276131 Karlsruhe

März 2010 